\title{
Prospects of heavy neutrino searches at future lepton colliders
}

\author{
Shankha Banerjee, ${ }^{1, *}$ P. S. Bhupal Dev ${ }^{2}, \dagger$ Alejandro

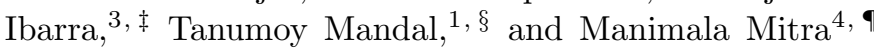 \\ ${ }^{1}$ Regional Centre for Accelerator-based Particle Physics, \\ Harish-Chandra Research Institute, Chhatnag Road, Jhusi, Allahabad 211019, India \\ ${ }^{2}$ Consortium for Fundamental Physics, School of Physics and Astronomy, \\ University of Manchester, Manchester M13 9PL, United Kingdom \\ ${ }^{3}$ Physik-Department T30d, Technische Univertität München, \\ James-Franck-Straße, D-85748 Garching, Germany \\ ${ }^{4}$ Institute for Particle Physics Phenomenology, \\ Durham University, Durham DH1 3LE, United Kingdom
}

We discuss the future prospects of heavy neutrino searches at next generation lepton colliders. In particular, we focus on the planned electron-positron colliders, operating in two different beam modes, namely, $e^{+} e^{-}$and $e^{-} e^{-}$. In the $e^{+} e^{-}$beam mode, we consider various production and decay modes of the heavy neutrino $(N)$, and find that the final state with $e+2 j+\not \mathbb{E}$, arising from the $e^{+} e^{-} \rightarrow N \nu$ production mode, is the most promising channel. However, since this mode is insensitive to the Majorana nature of the heavy neutrinos, we also study a new production channel $e^{+} e^{-} \rightarrow N e^{ \pm} W^{\mp}$, which leads to a same-sign dilepton plus four jet final state, thus directly probing the lepton number violation in $e^{+} e^{-}$colliders. In the $e^{-} e^{-}$beam mode, we study the prospects of the lepton number violating process of $e^{-} e^{-} \rightarrow W^{-} W^{-}$, mediated by a heavy Majorana neutrino. We use both cut-based and multivariate analysis techniques to make a realistic calculation of the relevant signal and background events, including detector effects for a generic linear collider detector. We find that with the cut-based analysis, the light-heavy neutrino mixing parameter $\left|V_{e N}\right|^{2}$ can be probed down to $\sim 10^{-4}$ at $95 \%$ C.L. for the heavy neutrino mass up to $400 \mathrm{GeV}$ or so at $\sqrt{s}=500$ $\mathrm{GeV}$ with $100 \mathrm{fb}^{-1}$ of integrated luminosity. For smaller mixing values, we show that a multivariate analysis can improve the signal significance by up to an order of magnitude. These limits will be at least an order of magnitude better than the current best limits from electroweak precision data, as well as the projected limits from $\sqrt{s}=14 \mathrm{TeV}$ LHC.

PACS numbers: 12.60.-i, 14.60.St, 12.38.Qk

Keywords: Beyond Standard Model, Neutrino Physics, $e^{+} e^{-}$Experiments

\footnotetext{
* shankha@hri.res.in

† bhupal.dev@hep.manchester.ac.uk

¥ alejandro.ibarra@ph.tum.de

$\S$ tanumoymandal@hri.res.in

ฯ manimala.mitra@durham.ac.uk
} 


\section{INTRODUCTION}

The observation of neutrino oscillations in solar, atmospheric, reactor and accelerator neutrino data (for a review, see e.g. [1]) has unequivocally established that at least two of the three active neutrinos have a non-zero mass and that individual lepton flavor is violated. This provides a conclusive experimental evidence for the existence of some new physics beyond the Standard Model (SM), and therefore, a precise understanding of the neutrino mass mechanism is an important step in unraveling the nature of new physics.

The minimal renormalizable extension of the SM to explain the observed smallness of neutrino masses is the neutrino portal, defined by the Lagrangian

$$
-\mathcal{L}=h_{\ell \alpha} \bar{L}_{\ell} \widetilde{\Phi} N_{\alpha}+\frac{1}{2} M_{N_{\alpha \beta}} \bar{N}_{\alpha}^{C} N_{\beta}+\text { H.c. }
$$

where $L_{\ell}=\left(\nu_{\ell}, \ell\right)_{L}^{\top}$ (with $\left.\ell=e, \mu, \tau\right)$ and $\Phi$ are the $S U(2)_{L}$ lepton and Higgs doublets respectively, $\widetilde{\Phi}=i \sigma_{2} \Phi^{*}\left(\sigma_{2}\right.$ being the second Pauli matrix) is the hypercharge conjugate of $\Phi, N_{\alpha}^{C} \equiv N_{\alpha}^{\top} C^{-1}(C$ being the charge conjugation matrix) denotes the charge conjugate field and $N_{\alpha}$ are SM gauge-singlet neutral fermions, usually known as the sterile neutrinos, since they can talk to the SM sector only via their mixing with the active neutrinos. In Eq. (1), $h_{\ell \alpha}$ are the dimensionless complex Yukawa couplings which, after the electroweak symmetry breaking, yield a Dirac mass matrix $M_{D}=h v, v$ being the Higgs vacuum expectation value. In addition, Eq. (1) allows a Majorana mass term $M_{N}$, since the sterile neutrinos carry no SM gauge charge. The existence of these new interactions in Eq. (1) leads to the following neutrino mass matrix in the flavor basis $\left\{\nu_{\ell}^{C}, N_{\alpha}\right\}$ :

$$
\mathcal{M}_{\nu}=\left(\begin{array}{cc}
0 & M_{D} \\
M_{D}^{\top} & M_{N}
\end{array}\right)
$$

The active-sterile neutrino mixing is parametrized by $\xi \sim M_{D} M_{N}^{-1}$. In our subsequent discussion, we often denote the elements of this matrix as $V_{\ell N}$ (see Section II for details). For $\|\xi\| \equiv \sqrt{\operatorname{Tr}\left(\xi^{\dagger} \xi\right)} \ll 1$, the light neutrino masses and mixing are given by the diagonalization of the effective mass matrix

$$
M_{\nu} \simeq-M_{D} M_{N}^{-1} M_{D}^{\top},
$$

whereas the heavy neutrino masses are of order $M_{N}$. This is known as the type-I seesaw mechanism [2-6].

From the above discussion, it is evident that there are two key aspects of the seesaw mechanism that can be probed experimentally: the Majorana mass $M_{N}$ of the sterile neutrinos and their mixing $V_{\ell N}$ with the active ones. The Majorana nature of both active and sterile neutrinos can in principle be probed via the lepton number violating (LNV) low-energy process of neutrinoless double beta decay $(0 \nu \beta \beta)$. However, an observation of $0 \nu \beta \beta$ does not necessarily probe the active-sterile mixing $V_{\ell N}$, since these mixing effects may not always give the dominant contribution over purely left-or right-handed contributions to the $0 \nu \beta \beta$ process; for a review, see e.g. [7]. Alternatively, a non-negligible value of $V_{\ell N}$ can be indirectly inferred from non-unitarity of the light neutrino mixing matrix [8,9] as well as in observables for lepton flavor violation (LFV) [10, 11], lepton non-universality and electroweak precision tests $[12-16]$. However, these low-energy observables by themselves do not prove the Majorana nature of neutrinos since models with pseudo-Dirac neutrinos can also give rise to large non-unitarity and LFV effects $[17-22]$.

On the other hand, the direct collider searches for heavy neutrinos at the energy frontier can provide a simultaneous probe of both the aspects of the seesaw mechanism, i.e. the Majorana nature of the heavy neutrinos $N$ and their mixing with the active neutrinos, provided the seesaw scale is accessible to the available center of mass energy; for a review, see e.g. [23]. ${ }^{1}$ In particular, electroweak-scale heavy neutrinos can be produced on-shell at colliders with a cross section depending on the strength of their mixing with the active neutrinos in the minimal setup, while their subsequent decay pattern is governed by whether they are Majorana or Dirac particles. At a hadron collider, the 'smoking gun' collider signal for heavy Majorana neutrinos is the same-sign dilepton final state with two jets and no missing transverse energy: $p p \rightarrow W^{*} \rightarrow N \ell^{ \pm} \rightarrow \ell^{ \pm} \ell^{ \pm} j j$ [41-46]. Using this channel, both CMS and ATLAS experiments at the LHC have set direct limits on the light-heavy neutrino mixing $\left|V_{\ell N}\right|^{2}$ (with $\ell=e, \mu$ ) for heavy neutrino masses between 100 and $500 \mathrm{GeV}$ [47-50]. With the run-II phase of the LHC with more energy

\footnotetext{
1 For some examples of low-scale seesaw models with relatively large active-sterile neutrino mixing, which lead to observable effects at colliders as discussed here, see [19, 24-40].
} 
and higher luminosity, and including the infrared enhancement effects due to $t$-channel photon-mediated processes [51], these limits could in principle be extended for $M_{N}$ up to $1 \mathrm{TeV}$ or so.

For electroweak-scale Dirac neutrinos, the same-sign dilepton signal is suppressed, and the 'smoking gun' signal at the LHC is the trilepton final state: $p p \rightarrow W^{*} \rightarrow N \ell^{ \pm} \rightarrow \ell^{ \pm} \ell^{\mp} \ell^{ \pm}+\mathbb{E}_{T}$ [52-57]. Analyzing the trilepton data from $\sqrt{s}=8 \mathrm{TeV}$ LHC [58], similar limits on $\left|V_{\ell N}\right|^{2}$ (with $\ell=e, \mu$ ) have been derived for $M_{N}$ up to $500 \mathrm{GeV}$ [57].

In light of vigorous discussions (see e.g. [59]) on the possibility of a future lepton collider, such as ILC [60], FCC-ee [61], CLIC [62], CEPC [63] and muon collider [64], it is worthwhile examining the prospects of heavy neutrino searches in the clean, almost background-free environment of a lepton collider. We recall that for heavy neutrino masses below the $Z$-boson threshold, using their possible production in the $Z$-boson decay $Z \rightarrow \nu_{\ell} N$ or $Z \rightarrow N \bar{\nu}_{\ell}$ [65], and its subsequent neutral current (NC) or charged current (CC) decays, 95\% C.L. limits on $\left|V_{\ell N}\right|^{2} \lesssim 10^{-5}$ were obtained by L3 [66] and DELPHI [67] collaborations from a reanalysis of the LEP data. A future high-luminosity $Z$-factory, such as the FCCee, will significantly enhance the sensitivity by several orders of magnitude [68]. Proposed fixed-target experiments such as SHiP [69] can also dramatically improve the limits on $\left|V_{\ell N}\right|^{2}$, though only in the low-mass range between 0.5 and $10 \mathrm{GeV}$ or so.

For heavy neutrino masses above the $Z$-boson threshold, a direct search was performed in $e^{+} e^{-}$ annihilation at LEP [70, 71], where a single heavy neutrino can be produced via its mixing with active neutrinos: $e^{+} e^{-} \rightarrow N \nu$ [72-77], and can subsequently decay via CC, NC or Yukawa interactions with the SM $W, Z$ or Higgs $(H)$ boson respectively: $N \rightarrow \ell^{-} W^{+}\left(\ell^{+} W^{-}\right), \nu(\bar{\nu}) Z, \nu(\bar{\nu}) H$. Concentrating on the decay channel $N \rightarrow e W$ with $W \rightarrow$ jets, which would lead to a single isolated electron plus hadronic jets and missing energy, the L3 collaboration put a 95\% C.L. upper limit on the mixing parameter $\left|V_{e N}\right|^{2}$ in a heavy neutrino mass range between 80 and $205 \mathrm{GeV}$ [71]. Similar limits were derived recently [16] using the ALEPH data on $e^{+} e^{-} \rightarrow W^{-} W^{+} \rightarrow \bar{\nu} \ell^{-} \ell^{+} \nu[78]$. However, these searches were kinematically limited by the maximum center-of-mass energy $\sqrt{s}=208 \mathrm{GeV}$ at LEP2. It is expected that future lepton colliders can significantly improve the sensitivity and extend the limits to higher heavy neutrino masses for both Dirac and Majorana cases. This is explicitly demonstrated here for the most promising channel $e^{+} e^{-} \rightarrow N \nu \rightarrow \ell j j \notin^{2}$ by performing a Monte Carlo simulation at $\sqrt{s}=350$ and $500 \mathrm{GeV}$ ILC, including realistic detector effects and background estimates. We have used both cut-based and multivariate analysis (MVA) techniques to derive the projected sensitivity limits for a generic ILC detector [79]. We find that a light-heavy mixing of $\left|V_{e N}\right|^{2}$ down to $\sim 10^{-4}$ can be probed at 95\% C.L. for the heavy neutrino mass up to $400 \mathrm{GeV}$ or so at $\sqrt{s}=500 \mathrm{GeV}$ ILC with $100 \mathrm{fb}^{-1}$ integrated luminosity. Although for concreteness, we have performed our simulations for the ILC benchmark parameters, this study is also relevant in the context of other future lepton and photon colliders [80].

Note that neither the existing LEP search channels $e^{+} e^{-} \rightarrow N \nu \rightarrow \ell j j \notin$ nor $e^{+} e^{-} \rightarrow W^{-} W^{+}$have LNV final states, and hence, it is not evident from these channels whether the heavy neutrinos are Majorana particles, as predicted by the usual seesaw mechanism. In principle, the Majorana or Dirac nature of the heavy neutrinos could be determined to some extent from the $e^{+} e^{-} \rightarrow N \nu \rightarrow \ell j j \notin$ mode using the distribution of the polar angle between the produced neutrino and the incoming electron [81]. Here, we propose an alternative clean way to determine the Majorana nature using a new LNV signal at $e^{+} e^{-}$colliders within the minimal seesaw:

$$
e^{+} e^{-} \rightarrow N e^{ \pm} W^{\mp} \rightarrow \ell^{ \pm} W^{\mp} e^{ \pm} W^{\mp} \rightarrow \ell^{ \pm} e^{ \pm}+4 j
$$

with same-sign dilepton plus four hadronic jets. Noting that the SM background for this process is extremely small, we perform a detailed detector-level simulation for the signal sensitivity and find that the detection of 10 signal events for a mixing parameter $\left|V_{e N}\right|=0.04$ would require an integrated luminosity of at least $700 \mathrm{fb}^{-1}$, which is within reach of next generation lepton colliders.

For completeness, we also present the heavy neutrino production cross sections for various other subdominant processes, such as the Higgsstrahlung process $e^{+} e^{-} \rightarrow Z H \rightarrow Z N \nu_{\ell}\left(\bar{\nu}_{\ell}\right)$ and the dominantly photon-mediated processes $e^{+} e^{-} \rightarrow N \ell^{ \pm} e^{\mp} \nu_{e}\left(\bar{\nu}_{e}\right)$. We could also have the processes $e^{ \pm} \gamma \rightarrow N W^{ \pm}$and $e^{ \pm} \gamma \rightarrow N \ell^{ \pm} \nu_{e}\left(\bar{\nu}_{e}\right)$, with a real photon coming from the electron or positron beam. These processes are of direct relevance for an electron-photon collider [82].

Finally, taking advantage of the fact that it is possible to switch the beam configuration of an $e^{+} e^{-}$ machine from $e^{+} e^{-}$to an $e^{-} e^{-}$mode [83], one can also look for an LNV signal in the process $e^{-} e^{-} \rightarrow$ $W^{-} W^{-} \rightarrow 4 j$ mediated by a $t$-channel Majorana neutrino [84-88]. We make a detailed detector-level analysis of this LNV signal and find that due to negligible SM background for this process, it offers

\footnotetext{
2 For a lepton collider, since the initial four-momenta of the particles are known, we can measure the full missing energy
} $\notin$ and not just the transverse component, as in the case of a hadron collider. 
an interesting alternative way to probe LNV in the electron sector, even though the cross section is suppressed by $\left|V_{e N}\right|^{4}$.

The plan of the paper is as follows: in Section II, we discuss various possible production modes of the heavy neutrino at a lepton collider. In Section III, we do a detailed event analysis using the cut-based techniques for the signal and background involving the final states with $e+2 j+\not$ and $e^{ \pm} e^{ \pm}+4 j$ at the $e^{+} e^{-}$collider and the LNV $4 j$ final state at the $e^{-} e^{-}$collider. In Section IV, we present an MVA to further enhance the signal sensitivity for the most promising channel with $e+2 j+\not$ final state. Our conclusions are given in Section V.

\section{HEAVY NEUTRINO PRODUCTION AT LEPTON COLLIDER}

In the minimal seesaw scenario, where the heavy neutrinos do not carry any gauge charge, the only way for them to communicate with the SM sector is through their mixing with the active neutrinos, as governed by the seesaw Lagrangian (1). To parametrize the light-heavy neutrino mixing, we first diagonalize the full neutrino mass matrix given in Eq. (2) by a unitary mixing matrix:

$$
\mathcal{V}^{\top} \mathcal{M}_{\nu} \mathcal{V}=\operatorname{diag}\left(m_{i}, M_{j}\right)
$$

where $m_{i}$ (with $i=1,2,3$ ) and $M_{j}$ (with $j=4,5, \ldots$ ) are respectively the light and heavy neutrino mass eigenvalues. The unitary matrix $\mathcal{V}$ has an exact representation in terms of a dimensionless matrix $\xi$ (which depends on $M_{D}$ and $M_{N}$ ): $[37,89]$

$$
\mathcal{V}=\left(\begin{array}{cc}
\left(\mathbf{1}+\xi^{*} \xi^{\top}\right)^{-1 / 2} & \xi^{*}\left(\mathbf{1}+\xi^{\top} \xi^{*}\right)^{-1 / 2} \\
-\xi^{\top}\left(\mathbf{1}+\xi^{*} \xi^{\top}\right)^{-1 / 2} & \left(\mathbf{1}+\xi^{\top} \xi^{*}\right)^{-1 / 2}
\end{array}\right)\left(\begin{array}{cc}
\mathcal{U} & \mathbf{0} \\
\mathbf{0} & \mathcal{U}^{\prime}
\end{array}\right)
$$

where $\mathcal{U}, \mathcal{U}^{\prime}$ are the unitary matrices diagonalizing the individual light and heavy neutrino mass matrices $M_{\nu}$ and $M_{N}$ respectively.

Now using Eqs. (5) and (6), the light neutrino flavor eigenstates $\nu_{\ell}$ can be related to the mass eigenstates $\widehat{\nu}_{i}$ and $\widehat{N}_{j}$ as follows:

$$
\nu_{\ell}=\left[\left(\mathbf{1}+\xi^{*} \xi^{\top}\right)^{-1 / 2}\right]_{\ell \ell^{\prime}} \mathcal{U}_{\ell^{\prime} i} \widehat{\nu}_{i}+\left[\xi^{*}\left(\mathbf{1}+\xi^{\top} \xi^{*}\right)^{-1 / 2}\right]_{\ell \ell^{\prime \prime}} \mathcal{U}_{\ell^{\prime \prime} j}^{\prime} \widehat{N}_{j} \equiv U_{\ell i} \widehat{\nu}_{i}+V_{\ell j} \widehat{N}_{j}
$$

where the first term on the right-hand side (RHS) measures the non-unitarity of the Pontecorvo-MakiNakagawa-Sakata mixing matrix, and the second term determines the size of the light-heavy neutrino mixing in $\mathrm{CC}$ and $\mathrm{NC}$ interactions involving neutrinos. In the charged-lepton mass diagonal basis, the $\mathrm{CC}$ interaction relevant for the production and decay of heavy neutrinos is given by

$$
-\mathcal{L}_{C C}=\frac{g}{\sqrt{2}} W_{\mu}^{-\bar{\ell} \gamma^{\mu}} P_{L} \nu_{\ell}+\text { H.c. }=\frac{g}{\sqrt{2}} W_{\mu}^{-\bar{\ell} \gamma^{\mu}} P_{L}\left(U_{\ell i} \widehat{\nu}_{i}+V_{\ell j} \widehat{N}_{j}\right)+\text { H.c. }
$$

where $P_{L}=\left(1-\gamma^{5}\right) / 2$ is the left-chirality projection operator. Similarly, the NC interaction is given by

$$
\begin{aligned}
-\mathcal{L}_{N C}=\frac{g}{2 \cos \theta_{w}} Z_{\mu} \bar{\nu}_{\ell} \gamma^{\mu} P_{L} \nu_{\ell}=\frac{g}{2 \cos \theta_{w}} & Z_{\mu}\left[\left(U^{\dagger} U\right)_{i j} \widehat{\bar{\nu}}_{i} \gamma^{\mu} P_{L} \widehat{\nu}_{j}+\left(V^{\dagger} V\right)_{i j} \widehat{\bar{N}}_{i} \gamma^{\mu} P_{L} \widehat{N}_{j}\right. \\
& \left.+\left\{\left(U^{\dagger} V\right)_{i j} \widehat{\bar{\nu}}_{i} \gamma^{\mu} P_{L} \widehat{N}_{j}+\text { H.c. }\right\}\right],
\end{aligned}
$$

where $\theta_{w}$ is the weak mixing angle. For our subsequent discussion, we use a model-independent phenomenological approach, parametrized by a single heavy neutrino mass scale $M_{N}$, assuming that any other heavy neutrinos present in the system are sufficiently heavy and do not affect our analysis. Henceforth, we denote the light-heavy mixing parameter simply as $V_{\ell N}$, without explicitly writing the heavy neutrino flavor index. This enables us to derive generic collider exclusion/sensitivity limits in the $\left(M_{N}, V_{\ell N}\right)$ parameter space, without referring to any particular low-scale seesaw model-building aspects.

From (8) and (9), we see that the cross section of heavy neutrino production in $e^{+} e^{-}$collision will be proportional to the mixing parameter $\left|V_{\ell N}\right|^{n}$, where $n=2$ or 4 , depending on the process considered (see below). Similarly, for the two-body decays of the heavy neutrino to the SM final states $\ell^{ \pm} W^{\mp}, Z \nu(\bar{\nu})$ and $H \nu(\bar{\nu})$ (if kinematically allowed), the corresponding partial decay widths are also proportional to 


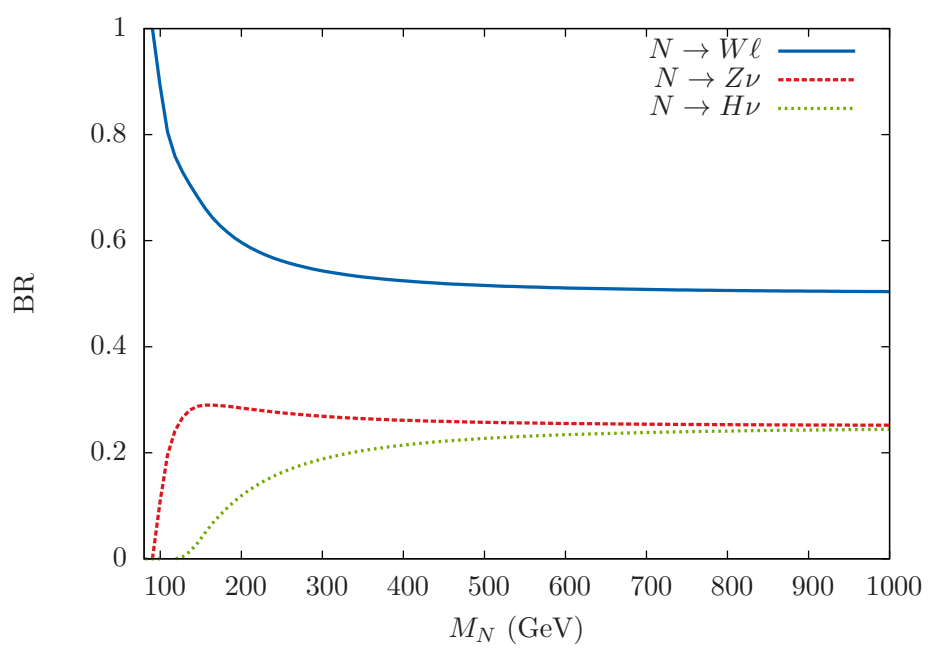

FIG. 1. The branching ratios (BR) of the heavy Majorana neutrino decay modes $N \rightarrow W^{ \pm} \ell^{\mp}, Z \nu(\bar{\nu}), H \nu(\bar{\nu})$ above the $Z$-boson threshold in the minimal seesaw model.

$\left|V_{\ell N}\right|^{2}:$

$$
\begin{aligned}
\Gamma\left(N \rightarrow \ell^{-} W^{+}\right) & =\frac{g^{2}}{64 \pi}\left|V_{\ell N}\right|^{2} \frac{M_{N}^{3}}{M_{W}^{2}}\left(1-\frac{M_{W}^{2}}{M_{N}^{2}}\right)^{2}\left(1+2 \frac{M_{W}^{2}}{M_{N}^{2}}\right), \\
\Gamma\left(N \rightarrow \nu_{\ell} Z\right) & =\frac{g^{2}}{128 \pi}\left|V_{\ell N}\right|^{2} \frac{M_{N}^{3}}{M_{W}^{2}}\left(1-\frac{M_{Z}^{2}}{M_{N}^{2}}\right)^{2}\left(1+2 \frac{M_{Z}^{2}}{M_{N}^{2}}\right), \\
\Gamma\left(N \rightarrow \nu_{\ell} H\right) & =\frac{g^{2}}{128 \pi}\left|V_{\ell N}\right|^{2} \frac{M_{N}^{3}}{M_{W}^{2}}\left(1-\frac{M_{H}^{2}}{M_{N}^{2}}\right)^{2} .
\end{aligned}
$$

Note that the total decay width for a heavy Majorana neutrino will be twice the sum of the partial decay widths shown in Eqs. (10)-(12), after taking into account the charge conjugate processes. The corresponding branching ratios (BR) are shown in Figure 1. For larger values of $M_{N}$, the branching ratios follow $\mathrm{BR}(\ell W): \operatorname{BR}(Z \nu): \operatorname{BR}(H \nu) \simeq 2: 1: 1$. Note that $\operatorname{BR}\left(N \rightarrow \ell^{-} W^{+}\right)$in the heavy Dirac neutrino case is twice as large compared to the heavy Majorana neutrino case, which has equal probability to decay into either $\ell^{-} W^{+}$or $\ell^{+} W^{-}$.

In what follows, we consider various heavy neutrino production channels at a lepton collider and numerically compute their production cross sections for given heavy neutrino mass and center of mass energy. ${ }^{3}$ For our subsequent collider analysis, we have implemented the relevant Lagrangian terms as shown in Eqs. (8) and (9) for heavy Majorana neutrino interactions in FEYNRuLEs2.0 [90], and generated the Universal FeynRules Output (UFO) [91] model files. These UFO model files have been used in the the Monte-CARLO (MC) event generator MADGraph5 [92] to generate the parton-level cross sections shown in this section, as well as the signal and background events for the analysis in Section III. The following basic trigger cuts on lepton transverse momentum $\left(p_{T}^{\ell}\right)$, pseudo-rapidity $\left(\eta^{\ell}\right)$ and radial distance $\left(\Delta R^{\ell \ell}\right)$ were used to identify the leptons in the final state:

$$
p_{T}^{\ell}>20 \mathrm{GeV}, \quad\left|\eta^{\ell}\right|<2.5, \quad \Delta R^{\ell \ell}>0.4 .
$$

For our numerical simulation, we consider two benchmark values for the center of mass energy $\sqrt{s}=350$ and $500 \mathrm{GeV}$. Also, we focus on heavy neutrino masses only above the $Z$-threshold. For $M_{N}<M_{Z}$, the existing LEP limits from $Z$-decay are quite stringent $[66,67]$ and one needs a high-luminosity $Z$ factory $[68,93]$ to significantly increase the sensitivity.

$$
\text { A. } e^{+} e^{-} \rightarrow N \nu \ell
$$

A single heavy neutrino of any flavor can be produced through an $s$-channel $Z$-exchange in $e^{+} e^{-}$ collisions, as shown in Figure 2 (a). ${ }^{4}$ The corresponding cross section will be proportional to $\left|V_{\ell N}\right|^{2}$. For

\footnotetext{
${ }^{3}$ For general analytic expressions for the production cross section, see [74].

${ }^{4}$ If $N$ is Majorana, both $\nu_{\ell}$ and $\bar{\nu}_{\ell}$ are allowed. However, for brevity, we denote both as simply $\nu_{\ell}$ in the following.
} 


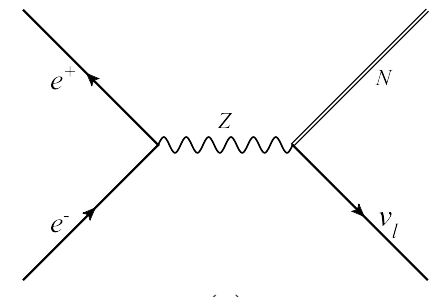

(a)

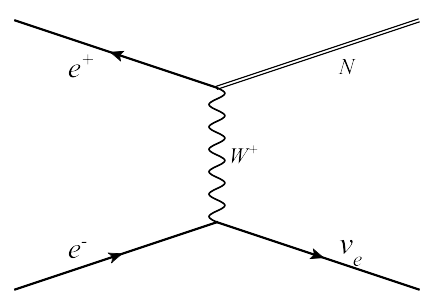

(b)

FIG. 2. (a) The $s$-channel and (b) $t$-channel Feynman diagrams for the process $e^{+} e^{-} \rightarrow N \nu_{\ell}$.

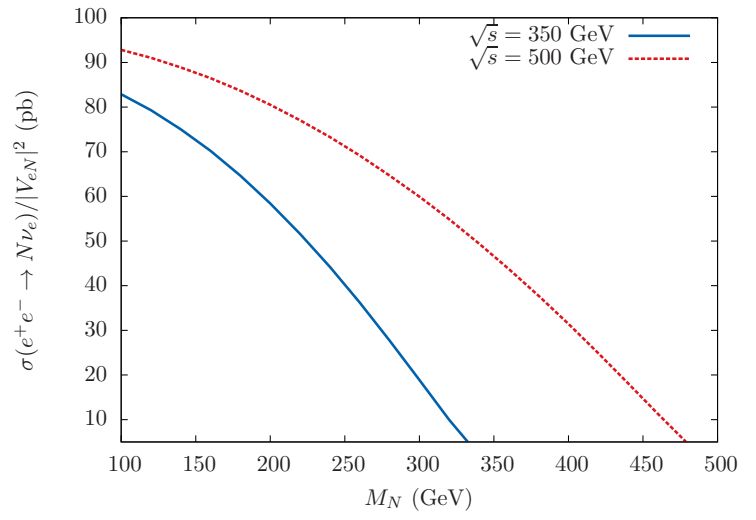

(a)

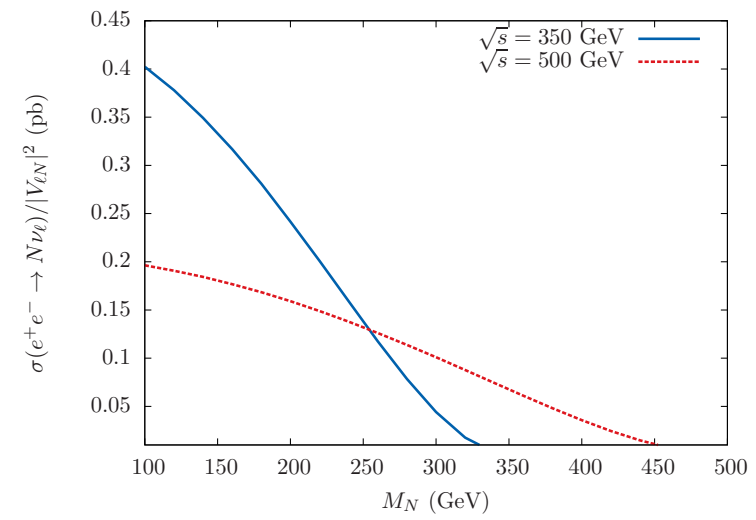

(b)

FIG. 3. Normalized heavy neutrino production cross section for the channel $e^{+} e^{-} \rightarrow \nu_{\ell} N$ for $\sqrt{s}=350$ and 500 $\mathrm{GeV}$. The left panel corresponds to $\ell=e$ (both $s$ and $t$ channels), whereas the right panel corresponds to $\ell=\mu, \tau$ (only the $s$ channel).

$\ell=e$, there is an additional $t$-channel $W$-exchange process, as shown in Figure 2 (b). The differential cross section for this process can be found in $[73,75]$. The numerical values of the total production cross sections are shown in Figure 3 for two different values of $\sqrt{s}=350$ and $500 \mathrm{GeV}$. The left panel of Figure 3 corresponds to the case $\ell=e$ and the right panel is for the case $\ell=\mu, \tau$.

The enhanced cross section in the electron channel is due to the additional $t$-channel contribution. Similarly, for the heavy neutrino decay, $N \rightarrow e W$ has the largest branching ratio, as evident from Figure 1. Thus, the channel $e^{+} e^{-} \rightarrow N \nu_{e}$, followed by $N \rightarrow e W$ and $W \rightarrow$ jets, has the largest signal cross section, and hence, can act as the most promising channel for the discovery of heavy neutrino mixing with electron neutrinos at a lepton collider. As a matter of fact, the existing direct search limit from LEP [71] comes from this channel.

Due to the relative smallness of the $s$-channel contribution, this production mode does not provide a very promising signal for probing $\left|V_{\ell N}\right|^{2}$ with $\ell=\mu, \tau$. Also note that the $s$-channel contribution to the cross section decreases with increasing $\sqrt{s}$, except when the heavy neutrino mass is close to the kinematic threshold, where the phase space suppression becomes more dominant. This is evident from Figure 3 (right panel). Using a suitable beam polarization might increase the signal sensitivity for $\ell=\mu, \tau$ [94], but this is of limited practical interest for a small $V_{e N}$.

We note here that since the hadronic activity at an $e^{+} e^{-}$collider is very limited, the background can be easily controlled with the help of simple kinematic cuts. Therefore, the other decay channels of the heavy neutrino, i.e. $N \rightarrow Z \nu_{\ell}, H \nu_{\ell}$, can also be used as complementary search channels, even though the corresponding BR are about a factor of two smaller than the $N \rightarrow W \ell$ mode. This will be illustrated in Section IV.

\section{B. $e^{+} e^{-} \rightarrow N \ell^{ \pm} W^{\mp}$}

The signal discussed in Section II A is insensitive to the Majorana nature of the heavy neutrino, and hence, does not probe LNV at a lepton collider. Here we present a new production mechanism $e^{+} e^{-} \rightarrow N \ell^{ \pm} W^{\mp}$ that can probe the Majorana nature of the heavy neutrinos at an $e^{+} e^{-}$collider. This is due to the fact that for a Majorana neutrino, both $\ell^{ \pm} W^{\mp}$ decay modes are allowed; hence, we get 


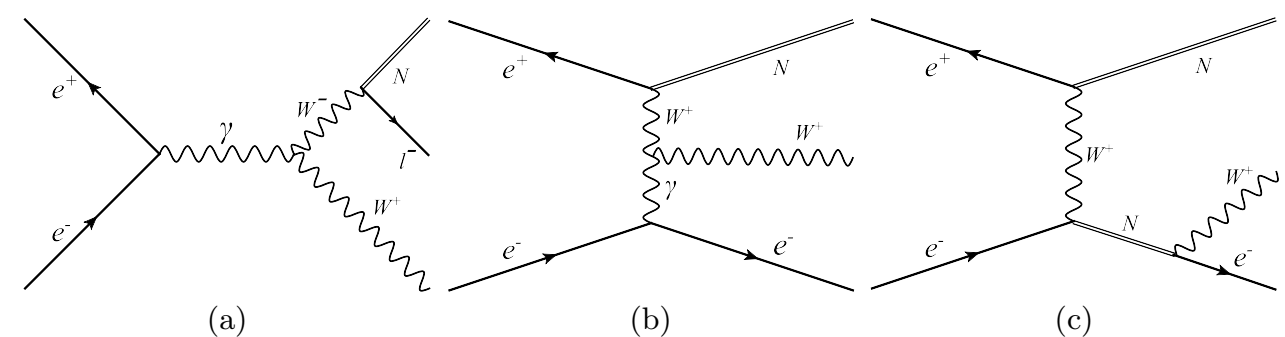

FIG. 4. Some sample Feynman diagrams for the process $e^{+} e^{-} \rightarrow N \ell^{-} W^{+}$, as discussed in Section IIB. The diagrams of type (a) can probe light-heavy neutrino mixing in all flavors, whereas the diagrams of type (b) and (c) can probe only the electron-sector mixing.

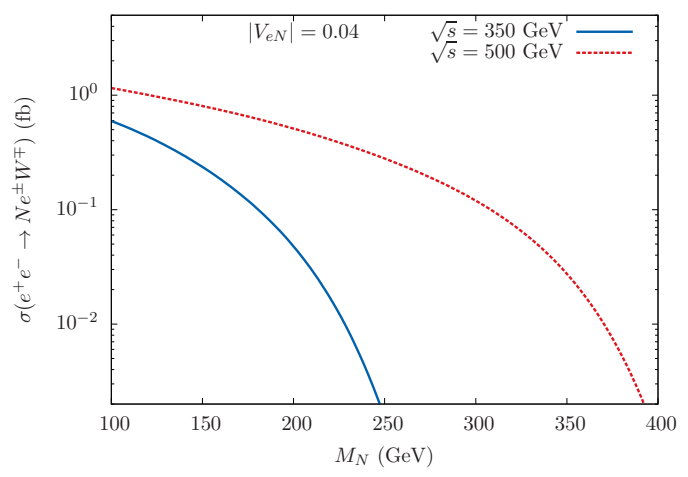

(a)

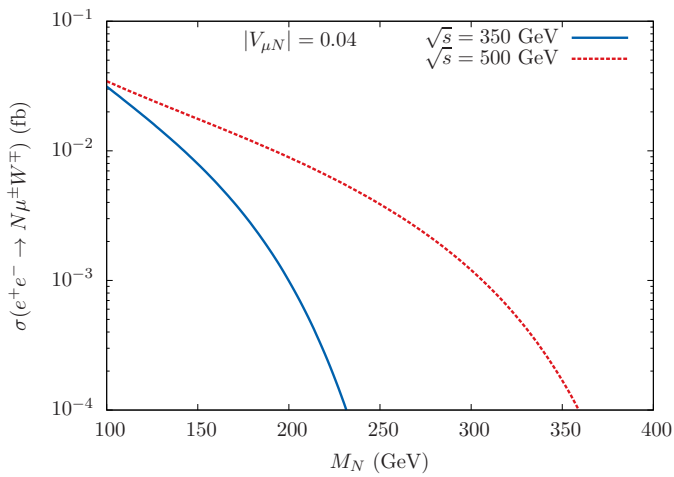

(b)

FIG. 5. Heavy neutrino production cross section for the channel $e^{+} e^{-} \rightarrow N \ell^{ \pm} W^{\mp}$ for $\sqrt{s}=350$ and $500 \mathrm{GeV}$, and with $\left|V_{\ell N}\right|=0.04$. The left panel is for mixing with electrons, whereas the right panel is for mixing with muon and tau sectors.

a LNV same-sign dilepton signal, along with two $W$ s. Thus, the dominant signal in this case will be $e^{ \pm} \ell^{ \pm}+4 j$ which probes LNV. In addition, for $\ell \neq e$, one can in principle also probe the LFV at future colliders.

This process gets contribution from several diagrams, and three typical diagrams are shown in Figure 4. For the electron sector, there is a dominant contribution from the $t$-channel photon diagrams [cf. Figure 4 (b)], thus leading to an infrared enhancement effect. ${ }^{5}$ This enhancement effect is absent in muon and tau sectors for an $e^{+} e^{-}$collider. The total production cross section for this process is given in Figure 5 . The left panel shows the cross section for electron-sector mixing and the right panel is for $\ell=\mu, \tau$. Note that this process involves diagrams with a heavy neutrino as as intermediate state [cf. Figure 4 (c)], and therefore, the total cross section for this process does not simply scale as $\left|V_{\ell N}\right|^{2}$, since the total heavy neutrino decay width in the propagator explicitly depends on $\left|V_{\ell N}\right|^{2}$. The cross section values shown in Figure 5 were obtained assuming a typical mixing parameter $\left|V_{\ell N}\right|=0.04$. A detailed detector-level simulation is done in Section III to derive the sensitivity for this process at future lepton colliders.

A related process to consider is $e^{ \pm} \gamma \rightarrow N W^{ \pm}$, where the real photon comes from one of the electron or positron beams. This process can be calculated using the Weizsacker-Williams equivalent photon approximation (EPA) [96, 97]. The results are shown in Figure 6. Again in this case, one can have a same-sign dilepton signal, when $N \rightarrow \ell^{ \pm} W^{\mp}$ and the other $W$ with the same sign as the lepton in the final state decays leptonically.

$$
\text { C. } e^{+} e^{-} \rightarrow N e^{ \pm} \ell^{\mp} \nu \ell
$$

Similar to the case discussed in Section II B, the process $e^{+} e^{-} \rightarrow N e^{ \pm} \ell^{\mp} \nu_{\ell}$ also contains infrared enhanced $t$-channel contributions mediated by virtual photons, as illustrated in Figure 7(a). The cross section for this process is given in Figure 8. Similar to the situation in Section II B, this process involves

\footnotetext{
5 This is similar to the infrared enhancement effects in hadron collisions: $p p \rightarrow N \ell^{ \pm} j j$ at the LHC [51, 57, 95].
} 


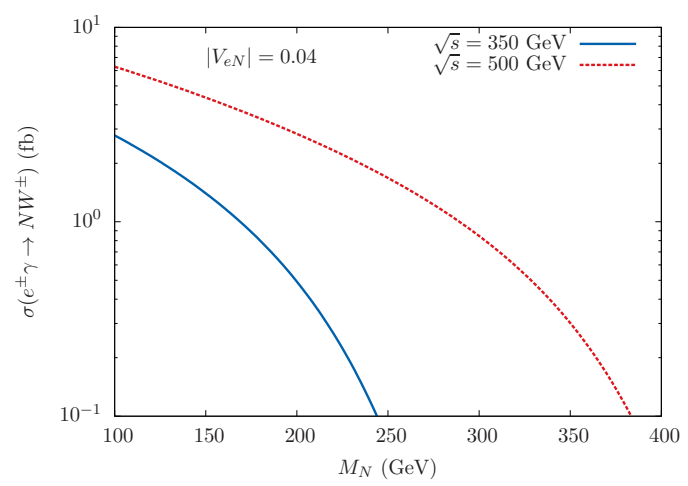

FIG. 6. Heavy neutrino production cross section for the channel $e^{ \pm} \gamma \rightarrow N W^{ \pm}$, with the real photon coming from one of the electron or positron beams, for $\sqrt{s}=350$ and $500 \mathrm{GeV}$, and with $\left|V_{e N}\right|=0.04$.

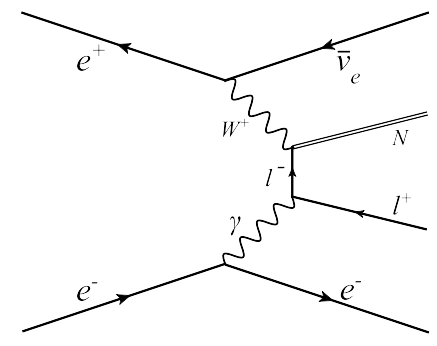

(a)

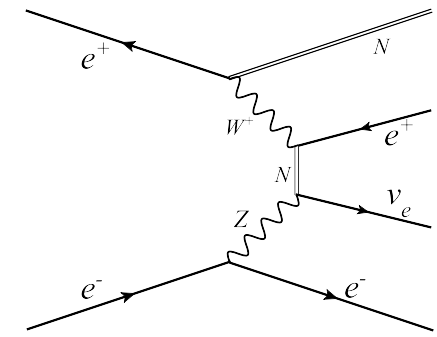

(b)

FIG. 7. Sample Feynman diagrams for the process $e^{+} e^{-} \rightarrow N e^{ \pm} \ell^{\mp} \nu$, as discussed in Section II C.

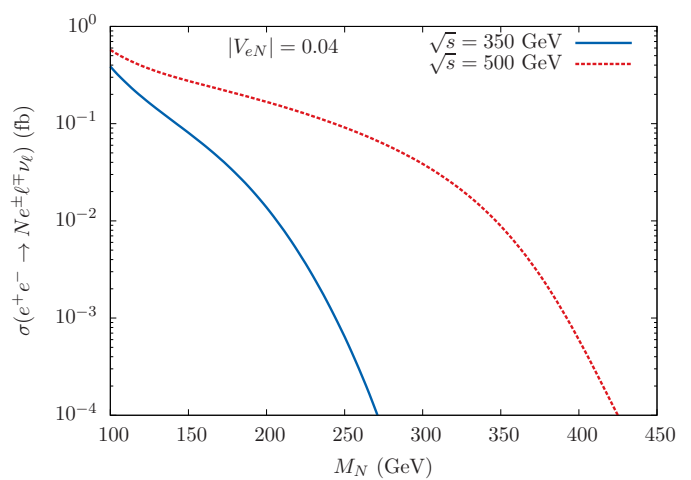

(a)

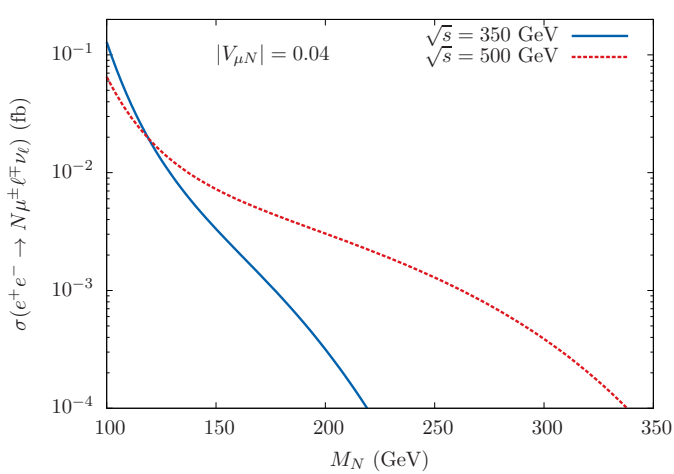

(b)

FIG. 8. Heavy neutrino production cross section for the channel $e^{+} e^{-} \rightarrow N \ell^{ \pm} \ell^{\prime \mp} \nu_{\ell}$ for $\sqrt{s}=350$ and $500 \mathrm{GeV}$, and with mixing $\left|V_{\ell N}\right|=0.04$. The left panel corresponds to $\ell=e$ and the right panel is for $\ell=\mu$, whereas $\ell^{\prime}$ could be any of the three lepton flavors.

Feynman diagrams with heavy neutrino propagators [cf. Figure 7(b)], and hence, the cross section does not simply scale as $\left|V_{\ell N}\right|^{2}$.

A related process to consider here is $e^{ \pm} \gamma \rightarrow N \ell^{ \pm} \nu_{\ell}$, where the real photon comes from one of the electron or positron beams. We calculate the cross section for this process using EPA and the results are shown in Figure 9.

\section{D. $e^{+} e^{-} \rightarrow Z H \rightarrow Z N \nu_{\ell}$}

This is the Higgsstrahlung process, followed by the decay of Higgs to $N \nu_{\ell}$, as shown in Figure 10. However, this process is only effective for $M_{N}$ values below the Higgs mass, as evident from the cross section values shown in Figure 11. This process can directly probe the heavy neutrino mixing with all neutrino flavors. Note that for the heavy neutrino mass in the vicinity of the Higgs mass, the Higgs 


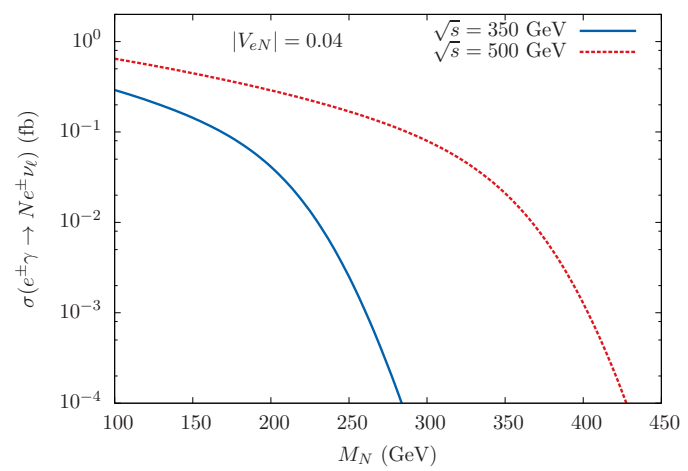

FIG. 9. Heavy neutrino production cross section for the channel $e^{ \pm} \gamma \rightarrow N \ell^{ \pm} \nu$, with the real photon coming from one of the electron or positron beams, for $\sqrt{s}=350$ and $500 \mathrm{GeV}$, and with $\left|V_{e N}\right|=0.04$.

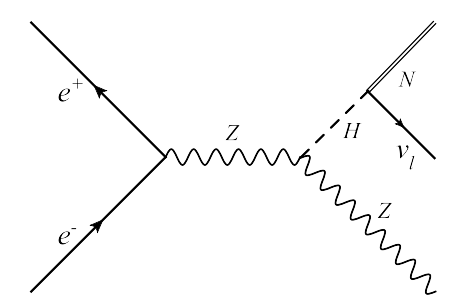

FIG. 10. Feynman diagram for the process $e^{+} e^{-} \rightarrow Z H \rightarrow Z N \nu_{\ell}$.

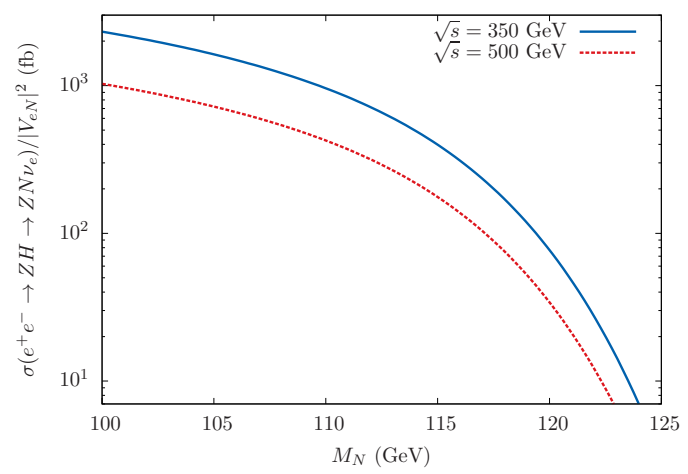

FIG. 11. Normalized heavy neutrino production cross section for the channel $e^{+} e^{-} \rightarrow Z N \nu_{\ell}$ for $\sqrt{s}=350$ and $500 \mathrm{GeV}$.

decay width will get significantly modified due to the new decay channels available, and using the Higgs signal strength data, one can also derive indirect constraints on the mixing parameter [98, 99].

E. $\quad e^{-} e^{-} \rightarrow W^{-} W^{-}$

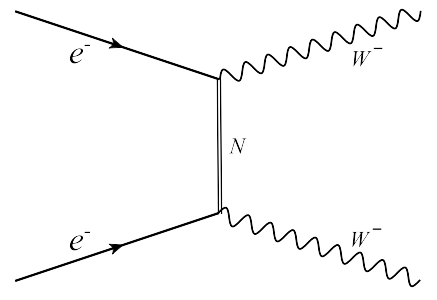

FIG. 12. Feynman diagram for the $t$-channel $N$ exchange. 


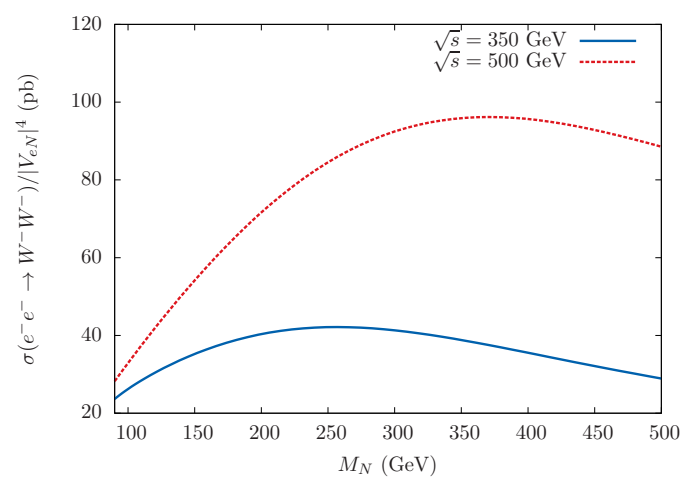

FIG. 13. Normalized cross section of the process $e^{-} e^{-} \rightarrow W^{-} W^{-}$for $\sqrt{s}=350$ and $500 \mathrm{GeV}$.

Experimentally, it is possible to switch from $e^{+} e^{-}$mode to the $e^{-} e^{-}$mode within the same experimental setup [83]. In this case, the Majorana nature of the heavy neutrino can be tested by observing the LNV process $e^{-} e^{-} \rightarrow W^{-} W^{-}$, as shown in Figure 12. This process is mediated by a $t$-channel $N$ exchange, ${ }^{6}$ and is free from SM background, except in the case when two additional leptons are produced and lost in the beam pipe. This can be considered as the lepton-collider analogue of the $0 \nu \beta \beta$ process. Moreover, for a linear collider like the ILC, it is also possible to use polarized electron beams. By using two left-handed electron beams, the cross-section for the $e^{-} e^{-} \rightarrow W^{-} W^{-}$channel will increase by a factor of four as compared to two unpolarized beams. On the other hand, for a circular collider like the FCC it is not possible to retain the polarization of the colliding beams due to the strong magnetic field used to bend the beam tracks.

The cross section for the channel shown in Figure 12 is proportional to $\left|V_{e N}\right|^{4}$ and the normalized cross section is shown in Figure 13 for $\sqrt{s}=350$ and $500 \mathrm{GeV}$. The produced $W$ s can decay either

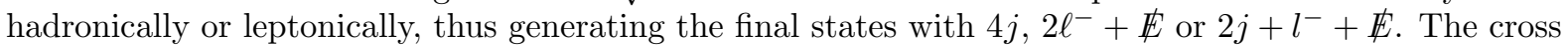
section values after the selection cuts and the signal sensitivity will be discussed in Section III.

\section{CUT-BASED ANALYSIS}

We have used the MC event generator MADGRAPH5 [92] to generate all signal and background events. The subsequent parton showering and hadronization have been done using PYTHIA6 [100]. We have used DeLPHES3 [101] for simplified detector simulation relevant for a generic $e^{+} e^{-}$collider. In this section, we present a cut-based analysis (CBA) to discriminate signal from the background and to derive the sensitivity limits for heavy neutrinos. In Section IV, we have employed a more sophisticated MVA for better signal-to-background discrimination, which leads to a better significance.

For our subsequent collider analysis, we apply the following basic trigger cuts, in addition to those in Eq. (13), to identify leptons and jets in the final state:

$$
p_{T}^{j}>20 \mathrm{GeV}, \quad\left|\eta^{j}\right|<2.5, \quad \Delta R^{j j}>0.4, \quad \Delta R^{\ell j}>0.4 .
$$

Jets are clustered using the anti- $k_{T}$ algorithm [102] implemented in FASTJET [103] with clustering parameter $R=0.4$. Then we apply further selection cuts for each signal discussed below, depending on its kinematics, in order to isolate it from the SM background, if present.

In the following, we consider various final states corresponding to the different heavy neutrino production modes, as discussed in Section II. Since the cross sections involving the mixing with the muon and tau sector are much smaller compared to the electron sector, we only consider the latter to derive the discovery prospects of the heavy neutrino at a future lepton collider.

$$
\text { A. } \quad e^{+} e^{-} \rightarrow N \nu_{e} \rightarrow e+2 j+\not
$$

The dominant SM background for this final state comes from the $e^{+} e^{-} \rightarrow W^{+} W^{-}$channel, followed by the leptonic decay of one $W$ and the hadronic decay of the other. Therefore, it is expected that

\footnotetext{
6 A similar diagram exists with a $t$-channel light Majorana neutrino exchange. However, its contribution to the cross section is extremely small, suppressed by the light neutrino mass.
} 


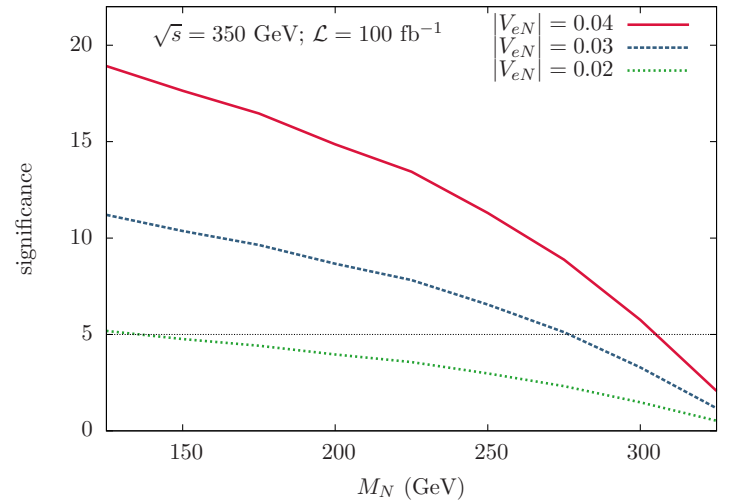

(a)

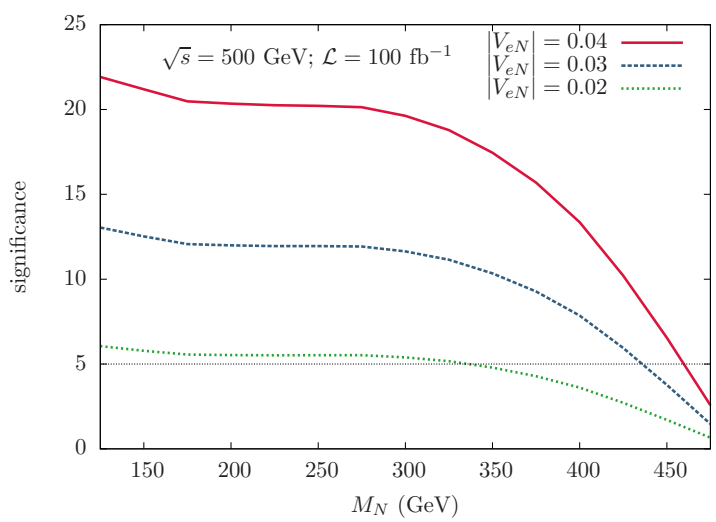

(b)

FIG. 14. Significance defined in Eq. (16) as functions of $M_{N}$ for different mixing values at (a) $\sqrt{s}=350 \mathrm{GeV}$ and (b) $\sqrt{s}=500 \mathrm{GeV}$, with $\mathcal{L}=100 \mathrm{fb}^{-1}$.

the invariant mass of the electron and the neutrino, viz. $M(e, \mathbb{E})$ peaks around the $W$-mass for the background, while for the signal, one does not expect such a behavior. On the other hand, the invariant mass distribution of the isolated electron and the two hardest tagged jets, $M\left(e, j_{1}, j_{2}\right)$, shows a peak near $M_{N}$ for the signal, but not for the background. These two distributions for the signal and background are shown later in Figure 18 in the context of a MVA. Therefore, $M(e, \notin)$ and $M\left(e, j_{1}, j_{2}\right)$ distributions can be used to separate the signal from the background in order to get a better sensitivity. Based on this observation, we demand the following selection cuts specific to this signal: exactly one isolated electron and at least two tagged jets satisfying the basic trigger cuts, no photon with $p_{T}>20 \mathrm{GeV}$ and

$$
\left|M\left(e, j_{1}, j_{2}\right)-M_{N}\right|<40 \mathrm{GeV}, \quad\left|M(e, \not)-M_{W}\right|>20 \mathrm{GeV} .
$$

\begin{tabular}{|c|c|c|c|c|c|}
\hline \multirow{2}{*}{$\begin{array}{c}M_{N} \\
(\mathrm{GeV})\end{array}$} & \multicolumn{2}{|c|}{ Signal (fb) } & \multicolumn{2}{|c|}{ Background (fb) } & $\mathcal{N}_{\mathcal{S}}$ \\
\hline & $\mathrm{TC}$ & $\mathrm{SC}$ & $\mathrm{TC}$ & $\mathrm{SC}$ & $\sqrt{\mathcal{N}_{\mathcal{S}}+\mathcal{N}_{\mathcal{B}}}$ \\
\hline 250 & 21.94 & 18.96 & 638.25 & 68.99 & 20.22 \\
\hline 350 & 16.33 & 13.48 & 638.25 & 46.11 & 17.46 \\
\hline
\end{tabular}

TABLE I. Signal $\left(\sigma_{\mathcal{S}}\right)$ and background $\left(\sigma_{\mathcal{B}}\right)$ cross sections after the trigger cuts (TC) and the selection cuts (SC) as defined in the text for different $M_{N}$ with $\left|V_{e N}\right|=0.04$ at $\sqrt{s}=500 \mathrm{GeV}$. The significance is computed using $100 \mathrm{fb}^{-1}$ integrated luminosity.

After imposing these selection criteria and including the detector effects, we compute the signal and background cross sections for two illustrative values of the heavy neutrino mass $M_{N}=250,350 \mathrm{GeV}$ at $\sqrt{s}=500 \mathrm{GeV}$ and for the mixing parameter $\left|V_{e N}\right|=0.04$. The results are shown in Table I. The signal significance

$$
n=\frac{\mathcal{N}_{\mathcal{S}}}{\sqrt{\mathcal{N}_{\mathcal{S}}+\mathcal{N}_{\mathcal{B}}}}
$$

where $\mathcal{N}_{\mathcal{S}}$ and $\mathcal{N}_{\mathcal{B}}$ are the number of signal and background events respectively, has been computed with an integrated luminosity of $100 \mathrm{fb}^{-1}$.

In Figure 14, we show the significance $n$ defined by Eq. (16) as a function of the heavy neutrino mass $M_{N}$, for different mixing values $\left|V_{e N}\right|=0.04,0.03$ and 0.02 at $\sqrt{s}=350$ and $500 \mathrm{GeV}$ for $\mathcal{L}=100$ $\mathrm{fb}^{-1}$. We see that mixing values up to $\left|V_{e N}\right|=0.02$ can be probed with a significance of $\gtrsim 5 \sigma$ for heavy neutrino mass $M_{N} \lesssim 350 \mathrm{GeV}$ at $\sqrt{s}=500 \mathrm{GeV}$.

In order to make a comparative study of the projected sensitivity obtained here with the existing and future limits from other contemporary experiments, we show the 95\% C.L. projected ILC limits on $\left|V_{e N}\right|^{2}$ as a function of $M_{N}$ in Figure 15. We have considered two configurations with $\sqrt{s}=350$ (green curves) and $500 \mathrm{GeV}$ (magenta curves) and with integrated luminosities $\mathcal{L}=100 \mathrm{fb}^{-1}$ (solid) and $500 \mathrm{fb}^{-1}$ (dashed). For larger $M_{N}$, the cross section decreases, as evident from Figure 3, thus leading to a weaker limit on $\left|V_{e N}\right|^{2}$. For comparison, we have also shown the current $95 \%$ C.L. direct exclusion limits from LEP 2 (red, shaded region) [71] and $\sqrt{s}=8 \mathrm{TeV}$ LHC data (blue, shaded region) [50], as well as a projected $95 \%$ C.L. limit from $\sqrt{s}=14 \mathrm{TeV}$ LHC with $300 \mathrm{fb}^{-1}$ integrated luminosity (blue, 


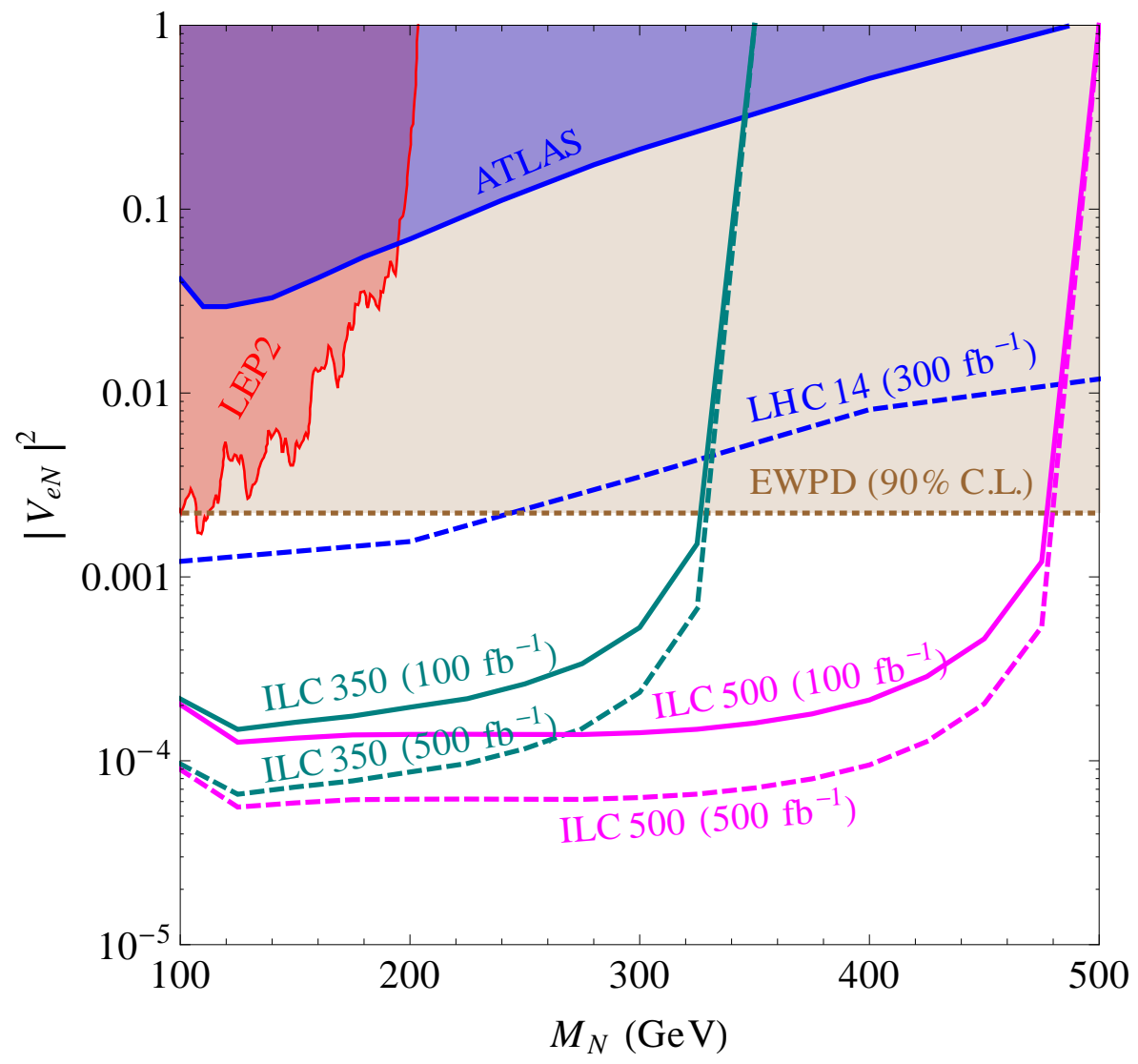

FIG. 15. The projected $95 \%$ C.L. ILC sensitivity of the light-heavy neutrino mixing parameter $\left|V_{e N}\right|^{2}$ as a function of the heavy neutrino mass $M_{N}$ for $\sqrt{s}=350$ (green) and $500 \mathrm{GeV}$ (magenta), and with $100 \mathrm{fb}^{-1}$ (solid) and $500 \mathrm{fb}^{-1}$ (dashed) integrated luminosity. Also shown are the current $90 \%$ C.L. indirect exclusion limit from electroweak precision data (EWPD; brown, dotted) [16], 95\% C.L. direct exclusion limits from LEP 2 (red, shaded) [71] and $\sqrt{s}=8 \mathrm{TeV}$ LHC data (blue, shaded) [50], as well as a projected 95\% C.L. limit from $\sqrt{s}=14 \mathrm{TeV}$ LHC with $300 \mathrm{fb}^{-1}$ integrated luminosity (blue, dashed) [23].

dashed curve) [23]. The best current limit is the indirect limit at 90\% C.L. (brown, dotted line) derived from an analysis of the EWPD [16]. ${ }^{7}$ As can be seen from Figure 15, a linear collider can significantly improve the heavy neutrino sensitivity up to mass values close to its kinematic threshold. Note that here we have not shown the constraints from $0 \nu \beta \beta$ (see e.g. [33, 36, 104-107]), since these limits are highly model dependent and can be significantly weakened in certain cases due to cancellation between different contributions [108].

As far as the other decay modes of the heavy neutrino are concerned, it can go to $Z \nu$ and $H \nu$ final states, followed by $Z \rightarrow \ell^{+} \ell^{-}, j j, H \rightarrow b \bar{b}$ and $W \rightarrow \ell \nu$ decay modes, thus giving rise to $j j+\not t$, $\ell^{+} \ell^{-}+\not \mathbb{E}, b \bar{b}+\not \mathbb{E}$ final states. For illustration, we show in Table II the signal cross sections of these final states for two benchmark values of $M_{N}$ at $\sqrt{s}=500 \mathrm{GeV}$. Note that the $2 \ell+\notin$ final state has two contributions coming from the leptonic decay of either $W$ or $Z$, as shown by the last two columns of Table II. Due to the relatively clean environment at a lepton collider, these processes can still lead to a non-negligible number of events with $500 \mathrm{fb}^{-1}$ luminosity. Note that for the channel involving Higgs decay, we have taken into account the correct Higgs decay width at next-to-next-to-leading order (NNLO): $\Gamma(H \rightarrow b \bar{b})_{\mathrm{NNLO}}=0.0023 \mathrm{GeV}[109]$, whereas the corresponding width obtained from MADGRAPH5 at leading order is $0.0053 \mathrm{GeV}$.

\footnotetext{
${ }^{7}$ For a detailed discussion of the present bounds on light-heavy neutrino mixing, see [23].
} 


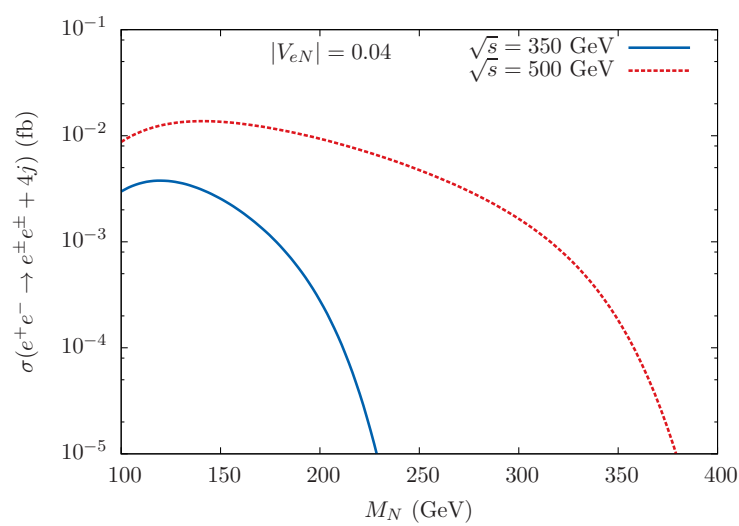

(a)

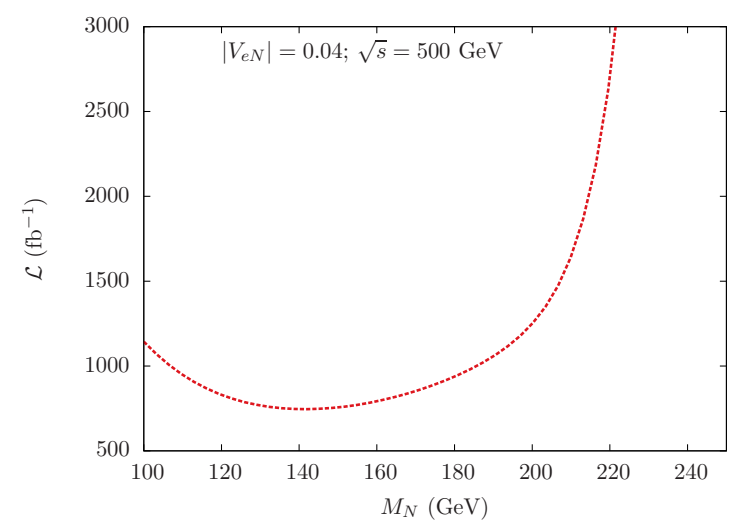

(b)

FIG. 16. (a) cross sections for the process $e^{+} e^{-} \rightarrow N e^{ \pm} W^{\mp} \rightarrow e^{ \pm} e^{ \pm}+4 j$ after trigger cuts and including detector efficiency at $\sqrt{s}=350,500 \mathrm{GeV}$ and (b) the required luminosity to observe 10 signal events with $\sqrt{s}=500 \mathrm{GeV}$. Here we have taken the mixing $\left|V_{e N}\right|=0.04$.

\begin{tabular}{|c|c|c|c|c|}
\hline$M_{N}(\mathrm{GeV})$ & $2 j+\not \mathbb{E}(\mathrm{fb})$ & $2 b+\not \mathbb{f}(\mathrm{fb})$ & $2 \ell+\not \mathbb{E}(Z)(\mathrm{fb})$ & $2 \ell+\not \mathbb{E}(W)(\mathrm{fb})$ \\
\hline 250 & 16.41 & 9.03 & 1.641 & 11.05 \\
\hline 400 & 7.415 & 5.20 & 0.7417 & 4.926 \\
\hline
\end{tabular}

TABLE II. Parton level signal cross sections for the subdominant decay modes of the heavy neutrino produced in the $e^{+} e^{-} \rightarrow N \nu_{\ell}$ process after trigger and selection cuts for illustrative cases of heavy neutrino masses $M_{N}=250$ and $400 \mathrm{GeV}$ and for mixing parameter $\left|V_{\ell N}\right|=0.04$.

\section{B. $e^{+} e^{-} \rightarrow N e^{ \pm} W^{\mp} \rightarrow e^{ \pm} e^{ \pm}+4 j$}

In this subsection, we consider the LNV process of $e^{+} e^{-} \rightarrow N e^{ \pm} W^{\mp} \rightarrow e^{ \pm} e^{ \pm}+4 j$, as discussed in Section IIB. We demand in the final state, two electrons (or two positrons) and at least four tagged jets satisfying the basic trigger cuts given in Eqs. (13) and (14). The signal cross section after imposing the cuts and including the detector efficiency is given in Figure 16 (a). The SM background for this process, mostly coming from $4 \ell+4 j$ final states with two leptons lost in the beam pipe, is negligible $\lesssim \mathcal{O}\left(10^{-5} \mathrm{fb}\right)$. Hence, we do not perform a detailed background simulation for this process. It is evident from Figure 16(a) that the signal cross section is also small $\left(<10^{-2} \mathrm{fb}\right)$ and drops sharply with increasing $M_{N}$. This makes it a challenging task to observe a reasonable number of signal events with $100 \mathrm{fb}^{-1}$ integrated luminosity. In Figure 16 (b), we show the required integrated luminosity for observing 10 signal events. We find that $\mathcal{L} \gtrsim 700 \mathrm{fb}^{-1}$ is required for this purpose at a $\sqrt{s}=500 \mathrm{GeV}$ ILC. This is nevertheless within reach of future lepton colliders [80]. Although this channel has a smaller cross section, as compared to the $e+2 j+\mathbb{E}_{T}$ channel discussed in Section III A, an observation of even a few LNV events in this channel will concretely establish the Majorana nature of heavy neutrinos, with profound implications on our understanding of the origin of neutrino mass.

$$
\text { C. } e^{-} e^{-} \rightarrow W^{-} W^{-} \rightarrow 4 j
$$

In $e^{+} e^{-}$colliders, the positron beam can be readily switched to an electron beam, thus converting to an $e^{-} e^{-}$machine. This freedom allows us to study LNV processes such as $e^{-} e^{-} \rightarrow W^{-} W^{-} \rightarrow 4 j$, as discussed in Section IIE. At the partonic level, there are no SM backgrounds for this LNV process. However, in realistic situations, different other processes can mimic the same final state as the signal. For instance, we can have $4 j+\not \mathbb{E}, 4 j+\not E+$ leptons or $4 j+$ leptons, where the additional leptons are lost in the beam pipe. The SM processes which contribute to the background are listed in Ref. [87]. We revisit these processes here and implicitly assume that we cannot reconstruct the charge of the gauge bosons from where the jets originated. The dominant background channels under consideration are : (P1) $e^{-} e^{-} \rightarrow W^{-} W^{-} \nu_{\ell} \nu_{\ell},(\mathrm{P} 2) e^{-} e^{-} \rightarrow W^{-} Z \nu_{\ell} e^{-},(\mathrm{P} 3) e^{-} e^{-} \rightarrow Z Z e^{-} e^{-}$and (P4) $e^{-} e^{-} \rightarrow W^{-} W^{+} e^{-} e^{-}$. All the gauge bosons decay hadronically so that we have four jets at the parton level. We shower the sample events and perform the detector simulation by demanding a minimum $\mathbb{E}_{T}$ of $20 \mathrm{GeV}$, no leptons 


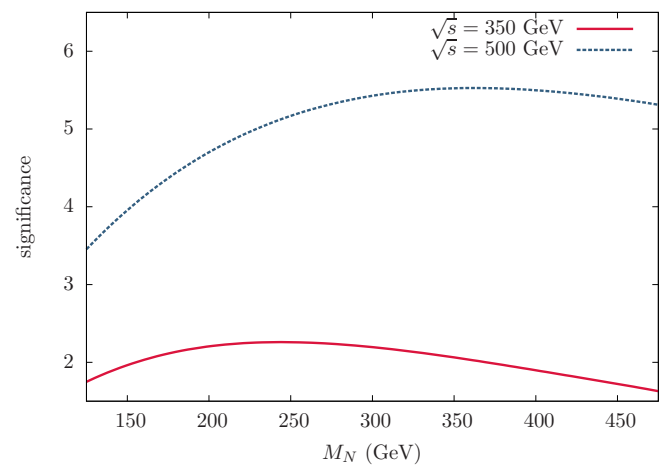

FIG. 17. The significance as a function of $M_{N}$ for the $e^{-} e^{-} \rightarrow 4 j$ channel for an integrated luminosity of 500 $\mathrm{fb}^{-1}$ at $\sqrt{s}=350,500 \mathrm{GeV}$.

and photons which pass the trigger cuts and at least 4 tagged jets satisfying the trigger cuts given in Eq. (14). The background cross sections before and after the selection cuts are shown in Table III. For $\sqrt{s}=500 \mathrm{GeV}$ and $\left|V_{e N}\right|=0.04$, we can achieve $>3 \sigma$ significance for the full range of the heavy neutrino mass considered here, as shown in Figure 17. However, given the smallness of the cross sections, the significance is somewhat lower at $\sqrt{s}=350 \mathrm{GeV}$. We comment here that a more involved search strategy using optimized cuts or a multivariate analysis might improve this significance. Also note that, in our analysis, we have used unpolarized electron beams. However, with the experimental facility at the ILC, it will be possible to use polarized beams which will lead to an increase in the cross sections by a factor of four.

\begin{tabular}{|c|c|c|c|c|c|c|c|c|c|}
\hline$\sqrt{s}$ & \multicolumn{2}{|c|}{$\sigma_{P 1}(\mathrm{fb})$} & \multicolumn{2}{|c|}{$\sigma_{P 2}(\mathrm{fb})$} & \multicolumn{2}{|c|}{$\sigma_{P 3}(\mathrm{fb})$} & \multicolumn{2}{|c|}{$\sigma_{P 4}(\mathrm{fb})$} & $\mathcal{N}_{B}$ \\
\cline { 2 - 9 }$(\mathrm{GeV})$ & TC & SC & TC & SC & TC & SC & TC & SC & \\
\hline 350 & 0.067 & 0.004 & 0.048 & 0.001 & $\sim 0$ & $\sim 0$ & 0.132 & 0.005 & 5 \\
\hline 500 & 0.453 & 0.015 & 0.399 & 0.004 & 0.006 & $\sim 0$ & 0.718 & 0.025 & 22 \\
\hline
\end{tabular}

TABLE III. Background cross sections for the signal process $e^{-} e^{-} \rightarrow W^{-} W^{-} \rightarrow 4 j$ after TC and SC for the four processes mentioned in the text for $\sqrt{s}=350 \mathrm{GeV}$ and $500 \mathrm{GeV}$. The number of background events $\mathcal{N}_{B}$ is computed at an integrated luminosity of $500 \mathrm{fb}^{-1}$.

For completeness, we also show the partonic cross sections for all possible decay modes of the $W$-pair in Table IV. Due to larger branching ratios for the hadronic decay of $W$, the $e^{-} e^{-} \rightarrow 4 j$ has the largest cross section. Note that for the numbers in Table IV, we have assumed the two $W$ s to be on-shell. The numbers for the second and fourth column will not change much if any of the $W$ s go off-shell; however, the numbers in the third column are valid only for on-shell $W$ s.

\begin{tabular}{|c|c|c|c|}
\hline$M_{N}(\mathrm{GeV})$ & $4 j(\mathrm{fb})$ & $2 \ell^{-}+\not \mathbb{E}(\mathrm{fb})$ & $\ell^{-}+2 j+\not \subset(\mathrm{fb})$ \\
\hline 250 & 0.075 & 0.009 & 0.055 \\
\hline 400 & 0.084 & 0.010 & 0.059 \\
\hline
\end{tabular}

TABLE IV. Parton level signal cross sections of different channels arising from $e^{-} e^{-} \rightarrow W^{-} W^{-}$after trigger and selection cuts for illustrative cases of the heavy neutrino masses $M_{N}=250$ and $400 \mathrm{GeV}$ and with mixing parameter $\left|V_{e N}\right|=0.04$ at $\sqrt{s}=500 \mathrm{GeV}$.

\section{MULTIVARIATE ANALYSIS}

In this section, we present a multivariate analysis for better signal-to-background discrimination, which leads to a better significance. For MVA, we use the Boosted Decision Tree (BDT) algorithm in the TMVA [110] framework. In the cut-based analysis for the channel $e^{+} e^{-} \rightarrow N \nu_{e} \rightarrow e+2 j+\mathbb{E}$ presented in Section III A, we have shown that two simple invariant mass cuts [cf. Eq. (15)] are sufficient to isolate the signal from the background with $\gtrsim 5 \sigma$ significance, particularly for larger mixing $\left(\left|V_{e N}\right| \gtrsim\right.$ $0.02)$ and in smaller mass range $(\lesssim 350 \mathrm{GeV})$. To probe smaller mixing $\left(\left|V_{e N}\right| \lesssim 0.02\right)$, we need to find an optimized set of cuts for the CBA in order to achieve $5 \sigma$ significance. For this purpose, the 
MVA technique is a useful tool to obtain the best sensitivity for a given set of parameters. For the MVA in the present case, we use nine variables, as listed in Table $\mathrm{V}$ with their relative importance in the MVA output response. These variables are chosen by comparing the signal trained for $M_{N}=250$ $\mathrm{GeV}$ with $\left|V_{e N}\right|=0.04$ at $\sqrt{s}=500 \mathrm{GeV}$ with the background distributions. Each of these variables has some reasonable amount of discriminating power, as shown in Figure 18, where we have plotted the normalized signal and background distributions. It is important to mention here that this set of nine variables used here might not be the optimal one and there is always a scope to improve the analysis with cleverer choices of variables. In our analysis, we have used these simple kinematic variables which are less correlated and have sufficiently good discriminating power. We use the MVA input-variables set optimized for the parameters $M_{N}=250 \mathrm{GeV},\left|V_{e N}\right|=0.04$ and $\sqrt{s}=500 \mathrm{GeV}$ for all parameter choices. In a more dedicated MVA, one can use different set of variables for different parameter points to further improve the analysis.

\begin{tabular}{|c|c|c|c|c|c|}
\hline Variable & Importance & Variable & Importance & Variable & Importance \\
\hline$p_{T}(e)$ & $9.123 \times 10^{-2}$ & $\left|\eta\left(j_{1}\right)\right|$ & $6.704 \times 10^{-2}$ & $\Delta R\left(e, j_{1}\right)$ & $5.662 \times 10^{-2}$ \\
\hline$|\eta(e)|$ & $7.824 \times 10^{-2}$ & $M(e, \not{E})$ & $1.527 \times 10^{-1}$ & $\Delta R\left(j_{1}, j_{2}\right)$ & $6.180 \times 10^{-2}$ \\
\hline$p_{T}\left(j_{1}\right)$ & $7.766 \times 10^{-2}$ & $M\left(e, j_{1}, j_{2}\right)$ & $2.522 \times 10^{-1}$ & Total $\not{E}$ & $1.625 \times 10^{-1}$ \\
\hline
\end{tabular}

TABLE V. Input variables used for MVA with $M_{N}=250 \mathrm{GeV},\left|V_{e N}\right|=0.04$ and $\sqrt{s}=500 \mathrm{GeV}$ and their relative importance. This might vary for other sets of parameters.
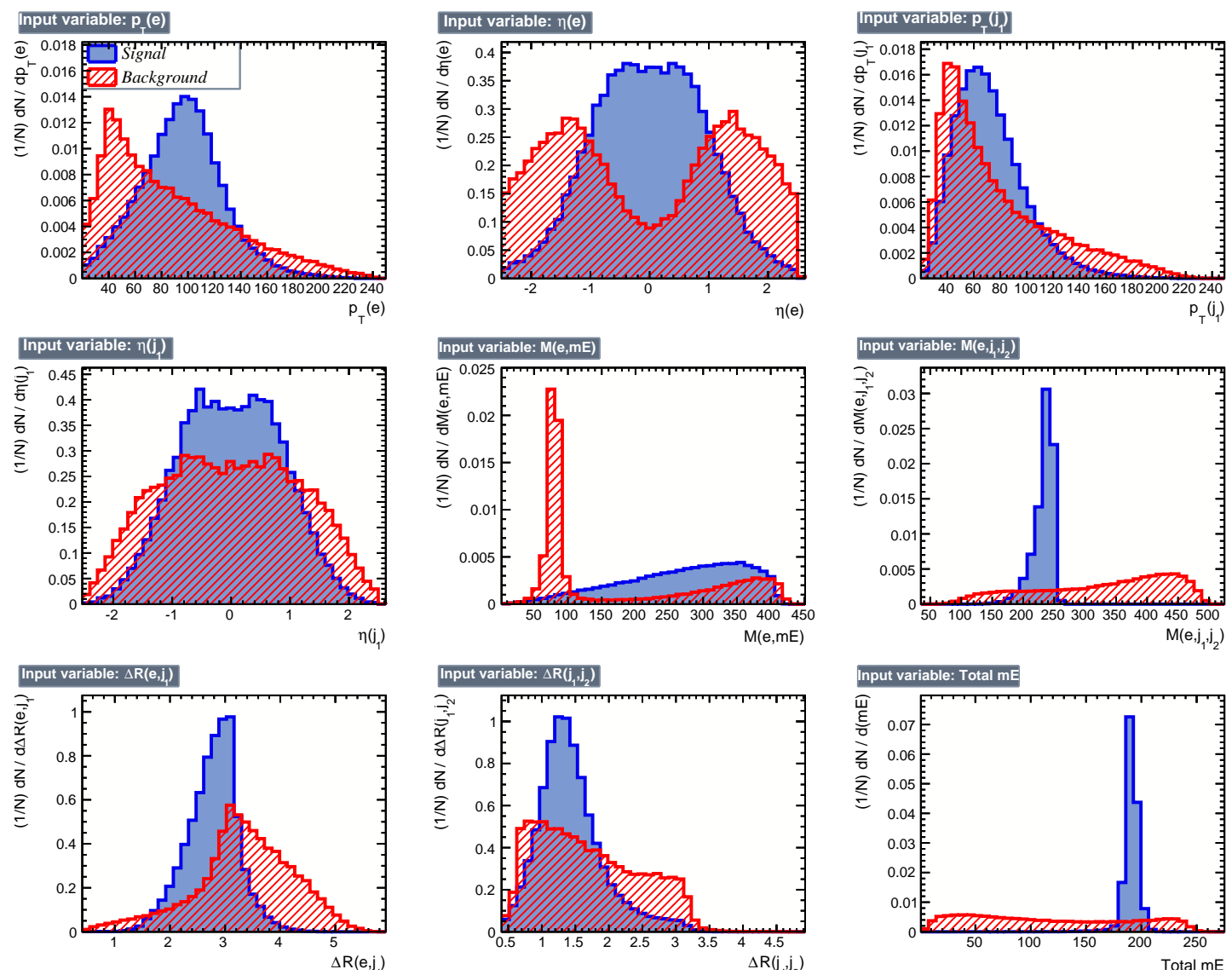

FIG. 18. Signal (blue) and background (red) distributions of the input variables used for MVA. All the distributions are drawn for $M_{N}=250 \mathrm{GeV},\left|V_{e N}\right|=0.04$ and $\sqrt{s}=500 \mathrm{GeV}$.

For MVA, we must always be very careful about overtraining the signal/background, since it can happen without the proper choices of the algorithm specific tuning parameters. Using the KolmogorovSmirnov (KS) test one can check whether a test sample is overtrained or not. Generally, the test sample is not overtrained if the KS probability lies within the range 0.1 to 0.9 . For most cases, a critical KS probability value greater than 0.01 [111] ensures that the samples are not overtrained. In Figure 19, we have shown the KS probability values for the signal and the background of the BDT response and this confirms that both signal and background samples are not overtrained. We have ensured that we 


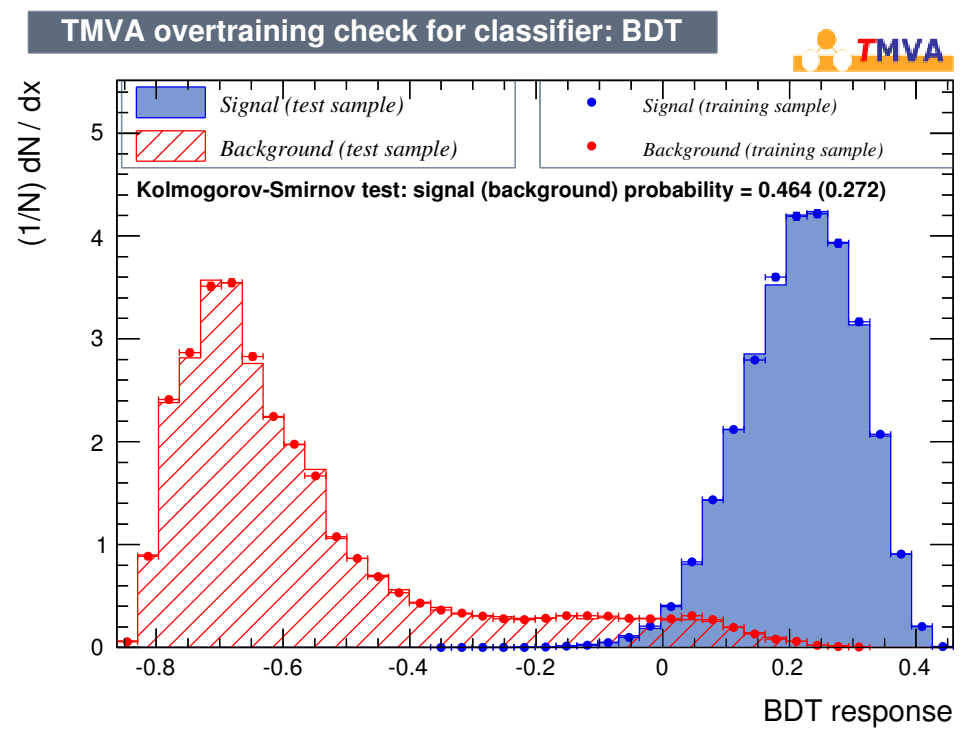

FIG. 19. BDT overtraining check for the parameter set $M_{N}=250 \mathrm{GeV}, V_{e N}=0.04$ and $\sqrt{s}=500$.

do not encounter overtraining for the full parameter range. We have used two statistically independent samples for MVA, one for training and the other for testing. As can be seen from Figure 19, the signal and background samples are well separated in this BDT output, and hence, using an appropriated BDT cut, we can significantly increase the signal significance.

In Table VI, we compute the significance for different $M_{N}$ and fixed $\left|V_{e N}\right|=0.04$ at $\sqrt{s}=500 \mathrm{GeV}$ with $\mathcal{L}=100 \mathrm{fb}^{-1}$. In Table VII, the significance is computed for different mixing values for a fixed $M_{N}=250 \mathrm{GeV}$ at $\sqrt{s}=500 \mathrm{GeV}$ with $\mathcal{L}=100 \mathrm{fb}^{-1}$. From these two tables, it is evident that MVA is more powerful to probe smaller mixing and larger masses where the performance of the CBA is comparatively weaker.

\begin{tabular}{|c|c|c|c|c|c|c|}
\hline $\begin{array}{c}M_{N} \\
(\mathrm{GeV})\end{array}$ & $\begin{array}{c}\sigma^{\text {w/o cut }} \\
(\mathrm{fb})\end{array}$ & $\begin{array}{c}\sigma^{\mathrm{TC}} \\
(\mathrm{fb})\end{array}$ & $\begin{array}{c}\text { BDT cut } \\
\text { value }\end{array}$ & $\mathcal{N}_{S}$ & $\mathcal{N}_{B}$ & $\frac{\mathcal{N}_{S}}{\sqrt{\mathcal{N}_{\mathcal{S}}+\mathcal{N}_{\mathcal{B}}}}$ \\
\hline 100 & 39.28 & 9.917 & 0.162 & 926 & 301 & 26.4 \\
\hline 150 & 38.40 & 22.61 & 0.162 & 1778 & 946 & 34.1 \\
\hline 200 & 34.06 & 22.73 & 0.159 & 1754 & 786 & 34.8 \\
\hline 250 & 30.22 & 21.94 & 0.156 & 1764 & 651 & 35.9 \\
\hline 300 & 25.79 & 19.87 & 0.136 & 1659 & 475 & 35.9 \\
\hline 350 & 20.42 & 16.33 & 0.167 & 1315 & 431 & 31.5 \\
\hline 400 & 13.95 & 11.43 & 0.232 & 876 & 294 & 25.6 \\
\hline 450 & 6.605 & 5.000 & 0.218 & 297 & 194 & 13.4 \\
\hline 475 & 2.815 & 2.361 & 0.280 & 119 & 357 & 5.5 \\
\hline \hline $\mathrm{SM}$ & 1022.5 & 638.3 & - & - & - & - \\
\hline
\end{tabular}

TABLE VI. Partonic cross sections $\left(\sigma^{\mathrm{w} / \mathrm{o}}{ }^{\mathrm{cut}}\right)$ and cross sections after TC $\left(\sigma^{\mathrm{TC}}\right)$ as defined in Section III A for the CBA with detector effects included for the process $e^{+} e^{-} \rightarrow N \nu_{e} \rightarrow e+2 j+\mathbb{E}$ at $\sqrt{s}=500 \mathrm{GeV}$ with mixing $\left|V_{e N}\right|=0.04$. Numbers of signal $\left(\mathcal{N}_{S}\right)$ and background $\left(\mathcal{N}_{B}\right)$ events for different $M_{N}$ after passing the BDT cut are shown for luminosity $\mathcal{L}=100 \mathrm{fb}^{-1}$.

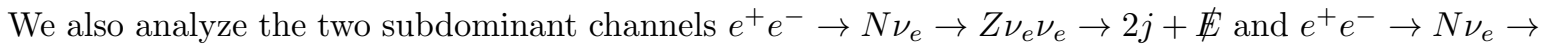
$H \nu_{e} \nu_{e} \rightarrow 2 b+\not E$ for a few illustrative masses using a simplified MVA. For these two channels we have used eight variables, viz. $p_{T}\left(j_{1}\right),\left|\eta\left(j_{1}\right)\right|, \mathbb{E}_{T},\left|\eta\left(\mathbb{E}_{T}\right)\right|, M\left(j_{1}, j_{2}\right), M\left(\notin, j_{1}\right), \Delta \eta\left(\notin, j_{1}\right)$ and scalar sum $H_{T}$ of the $p_{T}$ 's of all visible particles, to distinguish signal from background. Before the training and testing of the samples, they were made to pass the trigger cuts given in Section III A. In Table VIII for the channel $e^{+} e^{-} \rightarrow N \nu_{e} \rightarrow Z \nu_{e} \nu_{e} \rightarrow 2 j+\not ̈$, we show estimates for significance for three illustrative mass values, viz. $M_{N}=100,200$ and $300 \mathrm{GeV}$ for a fixed mixing $\left|V_{e N}\right|=0.04$ at $\sqrt{s}=350 \mathrm{GeV}$. Similarly, in Table IX we show analogous results for the channel $e^{+} e^{-} \rightarrow N \nu_{e} \rightarrow H \nu_{e} \nu_{e} \rightarrow 2 b+\not$ with mass values $M_{N}=150,200$ and $300 \mathrm{GeV}$ for a fixed mixing $\left|V_{e N}\right|=0.04$ at $\sqrt{s}=350 \mathrm{GeV}$. It is clear that using MVA, one can even probe these two subdominant channels with a good significance. It is important to note here that the significance for the process $e^{+} e^{-} \rightarrow N \nu_{e} \rightarrow H \nu_{e} \nu_{e} \rightarrow 2 b+\not E$ is subdued because of the 


\begin{tabular}{|c|c|c|c|c|c|c|}
\hline$\left|V_{\text {eN }}\right|$ & $\begin{array}{c}\sigma^{\text {w/o cut }} \\
(\mathrm{fb})\end{array}$ & $\begin{array}{c}\sigma^{\mathrm{TC}} \\
(\mathrm{fb})\end{array}$ & $\begin{array}{c}\text { BDT cut } \\
\text { value }\end{array}$ & $\mathcal{N}_{S}$ & $\mathcal{N}_{B}$ & $\frac{\mathcal{N}_{S}}{\sqrt{\mathcal{N}_{\mathcal{S}}+\mathcal{N}_{\mathcal{B}}}}$ \\
\hline 0.030 & 17.00 & 11.90 & 0.158 & 938 & 580 & 24.1 \\
\hline 0.025 & 11.81 & 8.27 & 0.201 & 582 & 360 & 19.0 \\
\hline 0.020 & 7.55 & 5.29 & 0.227 & 325 & 248 & 13.6 \\
\hline 0.015 & 4.25 & 2.98 & 0.220 & 187 & 303 & 8.4 \\
\hline 0.010 & 1.89 & 1.32 & 0.336 & 47 & 60 & 4.6 \\
\hline 0.0075 & 1.06 & 0.74 & 0.309 & 28 & 71 & 2.8 \\
\hline 0.0050 & 0.47 & 0.33 & 0.359 & 8 & 30 & 1.3 \\
\hline
\end{tabular}

TABLE VII. Same as Table VI for different mixing $\left|V_{e N}\right|$ and fixed $M_{N}=250 \mathrm{GeV}$.

smaller branching ratio of $N \rightarrow H \nu$ than that of $N \rightarrow Z \nu$ for the benchmark points under consideration (see Figure 1). Also we consider a single $b$-jet tagging in order to identify the Higgs, and this further reduces the signal due to the $b$-tagging efficiency, which could vary from about $40 \%$ for low $p_{T} b$-jets to about $70 \%$ for high $p_{T}(b)$. By demanding two $b$-tags, the significance will decrease even further. Once the experiments start running and the detector performances are better understood, one can try to look for cleverer variables to improve this significance.

\begin{tabular}{|c|c|c|c|c|c|c|}
\hline $\begin{array}{c}M_{N} \\
(\mathrm{GeV})\end{array}$ & $\begin{array}{c}\sigma^{\text {w/o cut }} \\
(\mathrm{fb})\end{array}$ & $\begin{array}{c}\sigma^{\mathrm{TC}} \\
(\mathrm{fb})\end{array}$ & $\begin{array}{c}\text { BDT cut } \\
\text { value }\end{array}$ & $\mathcal{N}_{S}$ & $\mathcal{N}_{B}$ & $\frac{\mathcal{N}_{S}}{\sqrt{\mathcal{N}_{\mathcal{S}}+\mathcal{N}_{\mathcal{B}}}}$ \\
\hline 100 & 8.27 & 5.89 & 0.026 & 480 & 8555 & 5.1 \\
\hline 200 & 13.61 & 10.22 & 0.017 & 724 & 6938 & 8.3 \\
\hline 300 & 4.39 & 3.738 & 0.082 & 275 & 5464 & 3.6 \\
\hline \hline $\mathrm{SM}$ & 290.0 & 218.9 & - & - & - & - \\
\hline
\end{tabular}

TABLE VIII. Same as Table VI for the process $e^{+} e^{-} \rightarrow N \nu_{e} \rightarrow Z \nu_{e} \nu_{e} \rightarrow 2 j+\not ̈$ at $\sqrt{s}=350 \mathrm{GeV}$.

\begin{tabular}{|c|c|c|c|c|c|c|}
\hline $\begin{array}{c}M_{N} \\
(\mathrm{GeV})\end{array}$ & $\begin{array}{c}\sigma^{\text {w/o cut }} \\
(\mathrm{fb})\end{array}$ & $\begin{array}{c}\sigma^{\mathrm{TC}} \\
(\mathrm{fb})\end{array}$ & $\begin{array}{c}\text { BDT cut } \\
\text { value }\end{array}$ & $\mathcal{N}_{S}$ & $\mathcal{N}_{B}$ & $\frac{\mathcal{N}_{S}}{\sqrt{\mathcal{N}_{\mathcal{S}}+\mathcal{N}_{\mathcal{B}}}}$ \\
\hline 150 & 2.24 & 0.795 & 0.243 & 36 & 260 & 2.1 \\
\hline 200 & 5.49 & 1.952 & -0.058 & 149 & 1580 & 3.6 \\
\hline 300 & 2.79 & 1.045 & -0.005 & 82 & 1621 & 2.0 \\
\hline \hline $\mathrm{SM}$ & 290.0 & 44.45 & - & - & - & - \\
\hline
\end{tabular}

TABLE IX. Same as Table VI for the process $e^{+} e^{-} \rightarrow N \nu_{e} \rightarrow H \nu_{e} \nu_{e} \rightarrow 2 b+\not t$ (only one $b$-jet is tagged among two $b$ 's) for c.m. energy $\sqrt{s}=350 \mathrm{GeV}$. The corresponding trigger cuts are defined in Section III A.

\section{CONCLUSION}

The seesaw mechanism provides a simple understanding of the observed smallness of the neutrino masses. Heavy Majorana neutrinos are an essential ingredient of the simplest seesaw scenario, and therefore, it is important to explore their experimental signatures in current and future experiments. In this work, we have studied the prospects of heavy neutrino searches at future lepton colliders. Due to their relatively cleaner environment, as compared to hadron colliders, lepton colliders provide us with a unique opportunity to probe the heavy neutrinos with higher sensitivity, which might shed light on the origin of neutrino mass and the associated new physics beyond the SM. In view of the increasingly hopeful case for an International Linear Collider in near future, we have mainly focused on various heavy neutrino production mechanisms at an $e^{+} e^{-}$collider and have performed a detailed detector-level simulation for the most promising channels. In particular, the production process $e^{+} e^{-} \rightarrow N \nu$ leading to the final state $e+2 j+\not$ has the largest cross section, and hence, we analyze this possibility in detail, taking into account realistic detector efficiency and SM background estimates. We have followed two different approaches to separate the signal from background. First, we present a conventional cut-based analysis using the distinct kinematic features of the signal over background. We show that in this approach, a light-heavy neutrino mixing parameter $\left|V_{e N}\right|^{2} \sim 10^{-4}$ can be probed at 95\% C.L. for a heavy neutrino mass up to $400 \mathrm{GeV}$ at $\sqrt{s}=500 \mathrm{GeV}$ ILC with luminosity of $100 \mathrm{fb}^{-1}$. Then we use a multivariate analysis with a boosted decision tree algorithm to make a better discrimination of the signal from background, which 
enables us to probe even smaller mixing values up to $\left|V_{e N}\right|^{2} \sim 10^{-5}$ or so for a larger range of heavy neutrino masses.

The dominant production mode $e^{+} e^{-} \rightarrow N \nu$ does not probe the Majorana nature of heavy neutrinos, which is a crucial aspect of the seesaw mechanism. To overcome this shortcoming at lepton colliders, we have studied a new production channel: $e^{+} e^{-} \rightarrow N \ell^{ \pm} W^{\mp}$, leading to a lepton number violating samesign dilepton plus four jet final state. Although the production cross section for this process is smaller than that of $e^{+} e^{-} \rightarrow N \nu$, the clean environment of $e^{+} e^{-}$colliders makes this process almost background free. Thus, any positive detection in this channel would be strong evidence for lepton number violation, with profound implications for our understanding of the neutrino mass mechanism. We find that a detection of 10 signal events in this channel for $\left|V_{e N}\right|=0.04$ would require an integrated luminosity of about $700 \mathrm{fb}^{-1}$ for $\sqrt{s}=500 \mathrm{GeV}$, which is certainly within reach of next generation lepton colliders.

Finally, we also study the lepton number violating process $e^{-} e^{-} \rightarrow W^{-} W^{-}$, mediated by a $t$-channel heavy neutrino exchange, followed by the hadronic decay of the $W$ s. This channel is again almost background-free. Hence, a positive observation of this signal will be an unambiguous probe of the Majorana nature of the heavy neutrinos. We show that in this channel, we can achieve $>3 \sigma$ significance for the full range of the heavy neutrino mass considered here at a $\sqrt{s}=500 \mathrm{GeV}$ ILC operating in the $e^{-} e^{-}$mode with a luminosity of $\mathcal{L}=500 \mathrm{fb}^{-1}$, provided the mixing is relatively large. With polarized beams, the signal cross section can be enhanced by a factor of four, thus leading to a better sensitivity.

Although our numerical simulations have been done specifically with the ILC in mind, our analysis can be easily extended to other next generation lepton colliders, such as the CLIC, FCC-ee, CEPC or even muon-muon, electron-photon and photon-photon colliders, once the design parameters and detector specifics become clearer and readily available for these machines.

\section{ACKNOWLEDGMENTS}

S.B. and T.M. thank Satyaki Bhattacharya and Shilpi Jain for their help regarding the multivariate analysis. P.S.B.D. thanks the local hospitality provided at TUM, Munich where part of this work was done. The work of S.B. and T.M. was partially supported by funding available from the Department of Atomic Energy, Government of India, for the Regional Centre for Accelerator-based Particle Physics (RECAPP), Harish-Chandra Research Institute. The work of P.S.B.D. is supported by the LancasterManchester-Sheffield Consortium for Fundamental Physics under STFC Grant No. ST/L000520/1. The work of A.I. was done in the context of the ERC Advanced grant project "FLAVOUR" (Grant No. 267104) and was partially supported by the DFG cluster of excellence "Origin and Structure of the Universe". M.M. acknowledges partial support of the ITN INVISIBLES (Marie Curie Actions, Grant No. PITN-GA-2011-289442).

[1] Particle Data Group Collaboration, K. A. Olive et al., Review of Particle Physics, Chin.Phys. C38 (2014) 090001.

[2] P. Minkowski, $\mu \rightarrow e \gamma$ at a Rate of One Out of 1-Billion Muon Decays?, Phys.Lett. B67 (1977) 421.

[3] R. N. Mohapatra and G. Senjanović, Neutrino mass and spontaneous parity nonconservation, Phys. Rev. Lett. 44 (1980) 912.

[4] T. Yanagida, Horizontal Symmetry And Masses Of Neutrinos, Conf.Proc. C7902131 (1979) 95.

[5] M. Gell-Mann, P. Ramond, and R. Slansky, Complex Spinors and Unified Theories, Conf.Proc. C790927 (1979) 315-321, [arXiv:1306.4669].

[6] J. Schechter and J. W. F. Valle, Neutrino masses in SU(2) $\times U(1)$ theories, Phys. Rev. D22 (1980) 2227.

[7] W. Rodejohann, Neutrino-less Double Beta Decay and Particle Physics, Int.J.Mod.Phys. E20 (2011) 1833, [arXiv:1106.1334].

[8] S. Antusch, C. Biggio, E. Fernandez-Martinez, M. Gavela, and J. Lopez-Pavon, Unitarity of the Leptonic Mixing Matrix, JHEP 0610 (2006) 084, [hep-ph/0607020].

[9] A. Abada, C. Biggio, F. Bonnet, M. B. Gavela, and T. Hambye, Low energy effects of neutrino masses, JHEP 12 (2007) 061, [arXiv:0707.4058].

[10] D. Dinh, A. Ibarra, E. Molinaro, and S. Petcov, The $\mu-e$ Conversion in Nuclei, $\mu \rightarrow e \gamma, \mu \rightarrow 3 e$ Decays and TeV Scale See-Saw Scenarios of Neutrino Mass Generation, JHEP 1208 (2012) 125, [arXiv:1205.4671].

[11] R. Alonso, M. Dhen, M. Gavela, and T. Hambye, Muon conversion to electron in nuclei in type-I seesaw models, JHEP 1301 (2013) 118, [arXiv:1209.2679].

[12] F. del Aguila, J. de Blas, and M. Perez-Victoria, Effects of new leptons in Electroweak Precision Data, Phys.Rev. D78 (2008) 013010, [arXiv:0803.4008].

[13] E. Akhmedov, A. Kartavtsev, M. Lindner, L. Michaels, and J. Smirnov, Improving Electro-Weak Fits with TeV-scale Sterile Neutrinos, JHEP 1305 (2013) 081, [arXiv:1302.1872]. 
[14] L. Basso, O. Fischer, and J. J. van der Bij, Precision tests of unitarity in leptonic mixing, Europhys.Lett. 105 (2014), no. 1 11001, [arXiv:1310.2057].

[15] S. Antusch and O. Fischer, Non-unitarity of the leptonic mixing matrix: Present bounds and future sensitivities, JHEP 1410 (2014) 94, [arXiv:1407.6607].

[16] S. Antusch and O. Fischer, Testing sterile neutrino extensions of the Standard Model at future lepton colliders, [arXiv:1502.05915].

[17] M. Malinsky, T. Ohlsson, and H. Zhang, Non-unitarity effects in a realistic low-scale seesaw model, Phys.Rev. D79 (2009) 073009, [arXiv:0903.1961].

[18] M. Malinsky, T. Ohlsson, Z.-z. Xing, and H. Zhang, Non-unitary neutrino mixing and CP violation in the minimal inverse seesaw model, Phys. Lett. B679 (2009) 242, [arXiv:0905.2889].

[19] P. S. B. Dev and R. N. Mohapatra, TeV Scale Inverse Seesaw in SO(10) and Leptonic Non-Unitarity Effects, Phys.Rev. D81 (2010) 013001, [arXiv:0910.3924].

[20] D. Forero, S. Morisi, M. Tortola, and J. W. F. Valle, Lepton flavor violation and non-unitary lepton mixing in low-scale type-I seesaw, JHEP 1109 (2011) 142, [arXiv:1107.6009].

[21] R. L. Awasthi and M. K. Parida, Inverse Seesaw Mechanism in Nonsupersymmetric SO(10), Proton Lifetime, Nonunitarity Effects, and a Low-mass Z' Boson, Phys.Rev. D86 (2012) 093004, [arXiv:1112.1826].

[22] P. Humbert, M. Lindner, and J. Smirnov, The Inverse Seesaw in Conformal Electro-Weak Symmetry Breaking and Phenomenological Consequences, [arXiv:1503.03066].

[23] F. F. Deppisch, P. S. B. Dev, and A. Pilaftsis, Neutrinos and Collider Physics, [arXiv:1502.06541].

[24] A. Pilaftsis, Radiatively induced neutrino masses and large Higgs neutrino couplings in the standard model with Majorana fields, Z.Phys. C55 (1992) 275, [hep-ph/9901206].

[25] W. Buchmuller, C. Greub, and P. Minkowski, Neutrino masses, neutral vector bosons and the scale of B-L breaking, Phys.Lett. B267 (1991) 395-399.

[26] J. Gluza, On teraelectronvolt Majorana neutrinos, Acta Phys.Polon. B33 (2002) 1735-1746, [hep$\mathrm{ph} / 0201002]$.

[27] A. Pilaftsis, Resonant tau-leptogenesis with observable lepton number violation, Phys.Rev.Lett. 95 (2005) 081602, [hep-ph/0408103].

[28] J. Kersten and A. Y. Smirnov, Right-Handed Neutrinos at CERN LHC and the Mechanism of Neutrino Mass Generation, Phys.Rev. D76 (2007) 073005, [arXiv:0705.3221].

[29] Z.-z. Xing, Naturalness and Testability of TeV Seesaw Mechanisms, Prog.Theor.Phys.Suppl. 180 (2009) 112, [arXiv:0905.3903].

[30] M. Gavela, T. Hambye, D. Hernandez, and P. Hernandez, Minimal Flavour Seesaw Models, JHEP 0909 (2009) 038, [arXiv:0906.1461].

[31] X.-G. He, S. Oh, J. Tandean, and C.-C. Wen, Large Mixing of Light and Heavy Neutrinos in Seesaw Models and the LHC, Phys.Rev. D80 (2009) 073012, [arXiv:0907.1607].

[32] R. Adhikari and A. Raychaudhuri, Light neutrinos from massless texture and below TeV seesaw scale, Phys.Rev. D84 (2011) 033002, [arXiv:1004.5111].

[33] A. Ibarra, E. Molinaro, and S. Petcov, TeV Scale See-Saw Mechanisms of Neutrino Mass Generation, the Majorana Nature of the Heavy Singlet Neutrinos and $(\beta \beta)_{0 \nu}$-Decay, JHEP 1009 (2010) 108, [arXiv:1007.2378].

[34] F. F. Deppisch and A. Pilaftsis, Lepton Flavour Violation and $\theta_{13}$ in Minimal Resonant Leptogenesis, Phys.Rev. D83 (2011) 076007, [arXiv:1012.1834].

[35] A. Ibarra, E. Molinaro, and S. T. Petcov, Low Energy Signatures of the TeV Scale See-Saw Mechanism, Phys.Rev. D84 (2011) 013005, [arXiv:1103.6217].

[36] M. Mitra, G. Senjanović, and F. Vissani, Neutrinoless Double Beta Decay and Heavy Sterile Neutrinos, Nucl.Phys. B856 (2012) 26, [arXiv:1108.0004].

[37] P. S. B. Dev and A. Pilaftsis, Minimal Radiative Neutrino Mass Mechanism for Inverse Seesaw Models, Phys.Rev. D86 (2012) 113001, [arXiv:1209.4051].

[38] P. S. B. Dev and A. Pilaftsis, Light and Superlight Sterile Neutrinos in the Minimal Radiative Inverse Seesaw Model, Phys.Rev. D87 (2013), no. 5 053007, [arXiv:1212.3808].

[39] C.-H. Lee, P. S. B. Dev, and R. N. Mohapatra, Natural TeV-scale left-right seesaw mechanism for neutrinos and experimental tests, Phys.Rev. D88 (2013) 093010, [arXiv:1309.0774].

[40] S. M. Boucenna, S. Morisi, and J. W. F. Valle, The low-scale approach to neutrino masses, AHEP 2014 (2014) 831598, [arXiv:1404.3751].

[41] W.-Y. Keung and G. Senjanovic, Majorana Neutrinos and the Production of the Right-Handed Charged Gauge Boson, Phys. Rev. Lett. 50 (1983) 1427.

[42] A. Datta, M. Guchait, and A. Pilaftsis, Probing lepton number violation via majorana neutrinos at hadron supercolliders, Phys.Rev. D50 (1994) 3195-3203, [hep-ph/9311257].

[43] T. Han and B. Zhang, Signatures for Majorana neutrinos at hadron colliders, Phys.Rev.Lett. 97 (2006) 171804, [hep-ph/0604064].

[44] S. Bray, J. S. Lee, and A. Pilaftsis, Resonant CP violation due to heavy neutrinos at the LHC, Nucl.Phys. B786 (2007) 95-118, [hep-ph/0702294].

[45] F. del Aguila, J. Aguilar-Saavedra, and R. Pittau, Heavy neutrino signals at large hadron colliders, JHEP 0710 (2007) 047, [hep-ph/0703261].

[46] A. Atre, T. Han, S. Pascoli, and B. Zhang, The Search for Heavy Majorana Neutrinos, JHEP 0905 (2009) 030, [arXiv:0901.3589]. 
[47] CMS Collaboration, S. Chatrchyan et al., Search for heavy Majorana neutrinos in $\mu^{+} \mu^{+}\left[\mu^{-} \mu^{-}\right]$and $e^{+} e^{+}\left[e^{-} e^{-}\right]$events in pp collisions at $\sqrt{s}=7$ TeV, Phys.Lett. B717 (2012) 109-128, [arXiv:1207.6079].

[48] CMS Collaboration, V. Khachatryan et al., Search for heavy Majorana neutrinos in $\mu^{ \pm} \mu^{ \pm}+$jets events in proton-proton collisions at $\sqrt{s}=8 \mathrm{TeV}$, [arXiv:1501.05566].

[49] ATLAS Collaboration, Search for Majorana neutrino production in pp collisions at $\sqrt{s}=7$ TeV in dimuon final states with the ATLAS detector, Tech. Rep. ATLAS-CONF-2012-139, CERN, Geneva, 2012.

[50] J. Klinger, Search for heavy Majorana neutrinos in pp collisions at $\sqrt{s}=8$ TeV with the ATLAS detector. $\mathrm{PhD}$ thesis, University of Manchester, 2014.

[51] P. S. B. Dev, A. Pilaftsis, and U.-K. Yang, New Production Mechanism for Heavy Neutrinos at the LHC, Phys.Rev.Lett. 112 (2014), no. 8 081801, [arXiv:1308.2209].

[52] F. del Aguila and J. Aguilar-Saavedra, Distinguishing seesaw models at LHC with multi-lepton signals, Nucl.Phys. B813 (2009) 22-90, [arXiv:0808.2468].

[53] F. del Aguila and J. Aguilar-Saavedra, Electroweak scale seesaw and heavy Dirac neutrino signals at LHC, Phys.Lett. B672 (2009) 158-165, [arXiv:0809.2096].

[54] F. del Aguila, J. Aguilar-Saavedra, and J. de Blas, Trilepton signals: the golden channel for seesaw searches at LHC, Acta Phys.Polon. B40 (2009) 2901-2911, [arXiv:0910.2720].

[55] C.-Y. Chen and P. S. B. Dev, Multi-Lepton Collider Signatures of Heavy Dirac and Majorana Neutrinos, Phys.Rev. D85 (2012) 093018, [arXiv:1112.6419].

[56] A. Das and N. Okada, Inverse seesaw neutrino signatures at the LHC and ILC, Phys.Rev. D88 (2013), no. 11 113001, [arXiv:1207.3734].

[57] A. Das, P. S. B. Dev, and N. Okada, Direct bounds on electroweak scale pseudo-Dirac neutrinos from $\sqrt{s}=8$ TeV LHC data, Phys.Lett. B735 (2014) 364-370, [arXiv:1405.0177].

[58] CMS Collaboration, S. Chatrchyan et al., Search for anomalous production of events with three or more leptons in pp collisions at $\sqrt{s}=8$ TeV, Phys.Rev. D90 (2014) 032006, [arXiv:1404.5801].

[59] R. Brock et al., Planning the Future of U.S. Particle Physics (Snowmass 2013): Chapter 3: Energy Frontier, [arXiv:1401.6081].

[60] ILC Collaboration, T. Behnke et al., The International Linear Collider Technical Design Report - Volume 1: Executive Summary, [arXiv:1306.6327].

[61] TLEP Design Study Working Group Collaboration, M. Bicer et al., First Look at the Physics Case of TLEP, JHEP 1401 (2014) 164, [arXiv:1308.6176].

[62] M. Aicheler et al., A Multi-TeV Linear Collider Based on CLIC Technology, . CERN-2012-007.

[63] A. Apyan et al., CEPC-SPPC Preliminary conceptual Design Report, . IHEP-AC-2015-001.

[64] MAP, MICE Collaborations, D. M. Kaplan, Muon Colliders and Neutrino Factories, [arXiv:1412.3487].

[65] M. Dittmar, A. Santamaria, M. Gonzalez-Garcia, and J. W. F. Valle, Production Mechanisms and Signatures of Isosinglet Neutral Heavy Leptons in $Z^{0}$ Decays, Nucl.Phys. B332 (1990) 1.

[66] L3 Collaboration, O. Adriani et al., Search for isosinglet neutral heavy leptons in Z0 decays, Phys.Lett. B295 (1992) 371-382.

[67] DELPHI Collaboration, P. Abreu et al., Search for neutral heavy leptons produced in $Z$ decays, Z.Phys. C74 (1997) 57-71.

[68] FCC-ee Study Team, A. Blondel, E. Gaverini, N. Serra, and M. Shaposhnikov, Search for Heavy Right Handed Neutrinos at the FCC-ee, [arXiv:1411.5230].

[69] W. Bonivento et al., Proposal to Search for Heavy Neutral Leptons at the SPS, [arXiv:1310.1762].

[70] L3 Collaboration, M. Acciarri et al., Search for Heavy Isosinglet Neutrinos in $e^{+} e^{-}$annihilation at $130<\sqrt{s}<189$ GeV, Phys.Lett. B461 (1999) 397-404, [hep-ex/9909006].

[71] L3 Collaboration, P. Achard et al., Search for heavy isosinglet neutrino in $e^{+} e^{-}$annihilation at LEP, Phys.Lett. B517 (2001) 67-74, [hep-ex/0107014].

[72] F. del Aguila, E. Laermann, and P. M. Zerwas, Exotic E(6) Particles in $e^{+} e^{-}$Annihilation, Nucl.Phys. B297 (1988) 1.

[73] W. Buchmuller and C. Greub, Heavy Majorana neutrinos in electron - positron and electron - proton collisions, Nucl.Phys. B363 (1991) 345-368.

[74] A. Djouadi, New fermions at $e^{+} e^{-}$colliders. 1. production and decay, Z. Phys. C63 (1994) 317-326, [hep$\mathrm{ph} / 9308339]$.

[75] G. Azuelos and A. Djouadi, New fermions at $e^{+} e^{-}$colliders. 2. Signals and backgrounds, Z.Phys. C63 (1994) 327-338, [hep-ph/9308340].

[76] J. Gluza and M. Zralek, Is there a chance to find heavy neutrinos in future linear colliders?, Phys.Lett. B372 (1996) 259-264, [hep-ph/9510407].

[77] J. Gluza, J. Maalampi, M. Raidal, and M. Zralek, Heavy neutrino mixing and single production at linear collider, Phys.Lett. B407 (1997) 45-52, [hep-ph/9703215].

[78] ALEPH Collaboration, A. Heister et al., Measurement of W-pair production in $e^{+} e^{-}$collisions at centreof-mass energies from 183-GeV to 209-GeV, Eur.Phys.J. C38 (2004) 147-160.

[79] T. Behnke et al., The International Linear Collider Technical Design Report - Volume 4: Detectors, arXiv:1306.6329.

[80] W. Barletta et al., Planning the Future of U.S. Particle Physics (Snowmass 2013): Chapter 6: Accelerator Capabilities, [arXiv:1401.6114].

[81] F. del Aguila and J. Aguilar-Saavedra, $\ell W \nu$ production at CLIC: A Window to TeV scale non-decoupled neutrinos, JHEP 0505 (2005) 026, [hep-ph/0503026]. 
[82] S. Bray, J. S. Lee, and A. Pilaftsis, Heavy Majorana neutrino production at $e^{-} \gamma$ colliders, Phys.Lett. B628 (2005) 250-261, [hep-ph/0508077].

[83] C. Adolphsen et al., The International Linear Collider Technical Design Report - Volume 3.II: Accelerator Baseline Design, [arXiv:1306.6328].

[84] J. Gluza and M. Zralek, On possibility of detecting the $e^{-} e^{-} \rightarrow W^{-} W^{-}$process in the standard model, Phys.Lett. B362 (1995) 148-154, [hep-ph/9507269].

[85] G. Belanger, F. Boudjema, D. London, and H. Nadeau, Inverse neutrinoless double beta decay revisited, Phys.Rev. D53 (1996) 6292-6301, [hep-ph/9508317].

[86] B. Ananthanarayan and P. Minkowski, CP violation in heavy neutrino mediated $e^{-} e^{-} \rightarrow W^{-} W^{-}$, Phys. Lett. B373 (1996) 130-134, [hep-ph/9512271].

[87] C. Greub and P. Minkowski, Heavy majorana neutrinos in $e^{-} e^{-}$collisions, eConf C960625 (1996) NEW149, [hep-ph/9612340].

[88] W. Rodejohann, Inverse Neutrino-less Double Beta Decay Revisited: Neutrinos, Higgs Triplets and a Muon Collider, Phys.Rev. D81 (2010) 114001, [arXiv:1005.2854].

[89] J. Korner, A. Pilaftsis, and K. Schilcher, Leptonic CP asymmetries in flavor changing $H^{0}$ decays, Phys. Rev. D47 (1993) 1080-1086, [hep-ph/9301289].

[90] N. D. Christensen and C. Duhr, FeynRules - Feynman rules made easy, Comput.Phys.Commun. 180 (2009) 1614-1641, [arXiv:0806.4194].

[91] C. Degrande, C. Duhr, B. Fuks, D. Grellscheid, O. Mattelaer, et al., UFO - The Universal FeynRules Output, Comput.Phys.Commun. 183 (2012) 1201-1214, [arXiv:1108.2040].

[92] J. Alwall, M. Herquet, F. Maltoni, O. Mattelaer, and T. Stelzer, MadGraph 5 : Going Beyond, JHEP 1106 (2011) 128, [arXiv:1106.0522].

[93] A. Abada, V. De Romeri, S. Monteil, J. Orloff, and A. Teixeira, Indirect searches for sterile neutrinos at a high-luminosity Z-factory, [arXiv:1412.6322].

[94] F. del Aguila, J. Aguilar-Saavedra, A. Martinez de la Ossa, and D. Meloni, Flavor and polarisation in heavy neutrino production at $e^{+} e^{-}$colliders, Phys.Lett. B613 (2005) 170-180, [hep-ph/0502189].

[95] D. Alva, T. Han, and R. Ruiz, Heavy Majorana Neutrinos from $W \gamma$ Fusion at Hadron Colliders, JHEP 1502 (2015) 072, [arXiv:1411.7305].

[96] C. von Weizsacker, Radiation emitted in collisions of very fast electrons, Z.Phys. 88 (1934) 612-625.

[97] E. Williams, Nature of the high-energy particles of penetrating radiation and status of ionization and radiation formulae, Phys.Rev. 45 (1934) 729-730.

[98] P. S. B. Dev, R. Franceschini, and R. N. Mohapatra, Bounds on TeV Seesaw Models from LHC Higgs Data, Phys.Rev. D86 (2012) 093010, [arXiv:1207.2756].

[99] C. G. Cely, A. Ibarra, E. Molinaro, and S. Petcov, Higgs Decays in the Low Scale Type I See-Saw Model, Phys.Lett. B718 (2013) 957-964, [arXiv:1208.3654].

[100] T. Sjostrand, S. Mrenna, and P. Z. Skands, PYTHIA 6.4 Physics and Manual, JHEP 05 (2006) 026, [hep-ph/0603175].

[101] DELPHES 3 Collaboration, J. de Favereau et al., DELPHES 3, A modular framework for fast simulation of a generic collider experiment, JHEP 1402 (2014) 057, [arXiv:1307.6346].

[102] M. Cacciari, G. P. Salam, and G. Soyez, The Anti- $k_{T}$ jet clustering algorithm, JHEP 0804 (2008) 063, [arXiv:0802.1189].

[103] M. Cacciari, G. P. Salam, and G. Soyez, FastJet User Manual, Eur.Phys.J. C72 (2012) 1896, [arXiv:1111.6097].

[104] M. Nemevsek, G. Senjanovic, and V. Tello, Connecting Dirac and Majorana Neutrino Mass Matrices in the Minimal Left-Right Symmetric Model, Phys.Rev.Lett. 110 (2013), no. 15 151802, [arXiv:1211.2837].

[105] P. S. B. Dev, S. Goswami, M. Mitra, and W. Rodejohann, Constraining Neutrino Mass from Neutrinoless Double Beta Decay, Phys.Rev. D88 (2013) 091301, [arXiv:1305.0056].

[106] P. S. B. Dev, S. Goswami, and M. Mitra, TeV Scale Left-Right Symmetry and Large Mixing Effects in Neutrinoless Double Beta Decay, [arXiv:1405.1399].

[107] A. Faessler, M. Gonzalez, S. Kovalenko, and F. Simkovic, Arbitrary mass Majorana neutrinos in neutrinoless double beta decay, Phys.Rev. D90 (2014) 096010, [arXiv:1408.6077].

[108] S. Pascoli, M. Mitra, and S. Wong, The Effect of Cancellation in Neutrinoless Double Beta Decay, Phys. Rev. D90 (2014), no. 9 093005, [arXiv:1310.6218].

[109] LHC Higgs Cross Section Working Group, S. Heinemeyer et al., Handbook of LHC Higgs Cross Sections: 3. Higgs Properties, [arXiv:1307.1347].

[110] A. Hocker, J. Stelzer, F. Tegenfeldt, H. Voss, K. Voss, et al., TMVA - Toolkit for Multivariate Data Analysis, PoS ACAT (2007) 040, [physics/0703039].

[111] D. Ciupke, Study of BDT Training Configurations with an Application to the $Z / H \rightarrow \tau \tau \rightarrow$ ee Analysis, http://www.desy.de/f/students/2012/reports/david_ciupke.pdf.gz, 2012. 